\title{
On the peripheral spectrum of positive elements
}

\author{
Egor A. Alekhno
}

\author{
Belarusian State University, Minsk, Belarus
}

\begin{abstract}
Let $A$ be an ordered Banach algebra with a unit e and a cone $A^{+}$. An element $p$ of $A$ is said to be an order idempotent if $p^{2}=p$ and $0 \leq p \leq \mathbf{e}$. An element $a \in A^{+}$is said to be irreducible if the relation $(\mathbf{e}-p) a p=0$, where $p$ is an order idempotent, implies $p=0$ or $p=\mathbf{e}$. For an arbitrary element $a$ of $A$ the peripheral spectrum $\sigma_{\text {per }}(a)$ of $a$ is the set $\sigma_{\text {per }}(a)=\{\lambda \in \mathbb{C}:|\lambda|=r(a)\}$, where $r(a)$ is the spectral radius of $a$. We investigate properties of the peripheral spectrum of an irreducible element $a$. The conditions under which $\sigma_{\text {per }}(a)$ contains of or coincides with $r(a) H_{m}$, where $H_{m}$ is the group of all $m^{\text {th }}$ roots of unity, and the spectrum $\sigma(a)$ is invariant under the rotation on angle $\frac{2 \pi}{m}$ for some $m \in \mathbb{N}$, are given. The correlation between these results and the existence of a cyclic form of $a$ is considered. The conditions under which $a$ is primitive, i.e., $\sigma_{\text {per }}(a)=\{r(a)\}$, are studied. The necessary assumptions on the algebra $A$ which imply the validity of these results, are discussed. In particular, the Lotz-Schaefer axiom is introduced and finite-rank elements of $A$ are defined. Other approaches to the notions of irreducibility and primitivity are discussed. The conditions under which the inequalities $0 \leq b<a$ imply $r(b)<r(a)$, are studied. The closedness of the center $A_{\mathbf{e}}$, i.e., of the order ideal generated by $\mathbf{e}$ in $A$, is proved.
\end{abstract}

Mathematical Subject Classification. 47A10, 46B40, 46H30, 46H10

Keywords. Ordered Banach algebra, Irreducible element, Peripheral spectrum, Primitivity, Lotz-Schaefer axiom, Finite-rank element, Center.

\section{Introduction and preliminaries}

Let $A$ be a (complex) Banach algebra with an algebraic unit e and $A^{+}$a (closed, convex) cone in $A$. As usual, for elements $a, b \in A$ the symbol $a \geq b$ (or $b \leq a$ ) means $a-b \in A^{+}$. Under this ordering, $A$ is an ordered linear space. From the definition of the cone, it follows that the inequalities $a \geq b$ and $b \geq a$ imply $a=b$ for all $a, b \in A$ and $\alpha x+\beta y \geq 0$ for all elements $x, y \in A^{+}$and all scalars $\alpha, \beta \in \mathbb{R}^{+}$. The elements of $A^{+}$are called positive. If $\mathbf{e} \geq 0$ and the inequalities $a, b \geq 0$ imply $a b \geq 0$ then $A$ is called an ordered Banach algebra. An important example of an ordered Banach algebra is the algebra of (linear, bounded) operators on an ordered Banach space. Namely, if $E$ is an ordered Banach space with (closed) cone $E^{+}$ then the algebra $B(E)$ of all operators on $E$ is an ordered Banach algebra under the natural order if and only if the linear space $E^{+}-E^{+}$is dense in $E$. In particular, if $E$ is a (complex) Banach lattice then the algebra $B(E)$ is an ordered Banach algebra.

The study of ordered Banach algebras was initiated in [13, 15]. In these papers and in a number of subsequent ones the main emphasis was on the study of spectral properties of positive elements. Nevertheless, in spite of the considerable progress in this direction, several aspects of the theory have received almost no attention. In particular, the properties of the peripheral spectrum of positive elements in ordered Banach algebras were not enough studied. The main purpose of this note is to take a step in this direction. 
We recall some well-known notions which will be necessary later on. Let $B$ be a Banach algebra with a unit. The spectrum [9, p. 19] of an element $b$ of $B$ is the set

$$
\sigma(b ; B)=\{\lambda \in \mathbb{C}: \lambda-b \text { is an invertible element of } B\} .
$$

When no confusion can occur, we write $\sigma(b)$ to denote $\sigma(b ; B)$. The resolvent set $\rho(b ; B)$ [1, p. 245] of $b$ is the complement of the spectrum, i.e., $\rho(b ; B)=\mathbb{C} \backslash \sigma(b ; B)$. As is well known, the spectrum $\sigma(b ; B)$ is a non-empty compact set (we assume $B \neq\{0\}$ ). The spectral radius $r(b)$ [9, p. 23] of an element $b$ is defined via the formula $r(b)=\max \{|\lambda|: \lambda \in \sigma(b ; B)\}$. By the Gelfand formula [9, pp. 11, 23], the equality $r(b)=\lim _{n \rightarrow \infty}\left\|b^{n}\right\|_{B}^{\frac{1}{n}}$ holds. The peripheral spectrum $\sigma_{\text {per }}(b ; B)$ (see, e.g., [13, Section 4]) of an element $b$ is the set

$$
\sigma_{\text {per }}(b ; B)=\{\lambda \in \sigma(b ; B):|\lambda|=r(b)\} .
$$

Obviously, $\sigma_{\text {per }}(b ; B)$ is also a non-empty compact set. Next, the resolvent function [1, p. 245] $R(\cdot, b): \rho(b ; B) \rightarrow B$ of $b$ is defined by $R(\lambda, b)=(\lambda-b)^{-1}$ and is an analytic function on the open set $\rho(b ; B)$. In particular, if $\lambda_{0}$ is an isolated point of $\sigma(b ; B)$ then in a sufficiently small punctured neighbourhood of this point a Laurent series expansion

$$
R(\lambda, b)=\sum_{j=-\infty}^{+\infty} b_{\lambda_{0}, j}\left(\lambda-\lambda_{0}\right)^{j}
$$

holds, where $b_{\lambda_{0}, j} \in B$ for all $j \in \mathbb{Z}$. Of course, this expansion also holds when the point $\lambda_{0} \notin \sigma(b ; B)$; in this case, $b_{\lambda_{0}, j}=0$ for $j \leq-1$. If $\lambda_{0}=r(a)$, we will simply write $b_{j}$ instead of $b_{\lambda_{0}, j}$. A point $\lambda_{0}$ is said to be a pole [1, p. 264] of the resolvent $R(\cdot, b)$ of order $k \in \mathbb{N}$ whenever $\lambda_{0}$ is an isolated point of $\sigma(b ; B), b_{\lambda_{0}, j}=0$ for $j<-k$, and $b_{\lambda_{0},-k} \neq 0$; in this case, the identities $b b_{\lambda_{0},-k}=b_{\lambda_{0},-k} b=\lambda_{0} b_{\lambda_{0},-k}$ hold. If $k=1$ then $\lambda_{0}$ is called a simple pole.

The considerable progress in the study of the peripheral spectrum was attained in the case of ordered spaces. The results concerning to the spectral theory of non-negative matrices, i.e., to the spectral theory in finite dimensional (Archimedean) Riesz spaces, and, in particular, to properties of the peripheral spectrum of non-negative matrices can be found in [16, Chapter 1] and [1, Chapter 8]. Properties of the peripheral spectrum of positive operators on Banach lattices are considered, e.g., in [16, Chapter V and, in particular, Sections V.4 and V.5] (see also [2, 1, 11]). Some results concerning to the peripheral spectrum of positive elements in ordered Banach algebras can be found in [13, Section 4]. Below, as far as necessary, we recall some results in these directions. Now we mention only the following result [15]. If $A$ is an ordered Banach algebra such that the spectral radius function in $A$ is monotone, i.e., the inequalities $0 \leq a \leq b$ in $A$ imply $r(a) \leq r(b)$, then $r(a) \in \sigma_{\text {per }}(a)$; in particular, this is true when the cone $A^{+}$is normal and, hence, when the ordered linear space $A$ is Dedekind complete.

The paper is organized as follows. In the second section properties of the peripheral spectrum of irreducible elements are studied (see, in particular, Theorem 12) and some assumptions on the algebra $A$ which allow obtaining nice spectral properties, are considered. In the next section, using a special assumption (the Lotz-Schaefer axiom), the results of the preceding section will be made more precisely and, moreover, the notion of finite-rank elements in ordered Banach algebras and the conditions of the primitivity of irreducible elements are discussed. 
Another approach to the notion of irreducibility is considered in the fourth section. In the next section, conditions under which the inequalities $0 \leq b<a$ imply $r(b)<r(a)$ are studied. In the last section, the closedness of the center $A_{\mathbf{e}}$ of an ordered Banach algebra $A$ is proved.

For any unexplained terminology, notations, and elementary properties of ordered Banach spaces, we refer the reader to [7]. For information on the theory of Riesz spaces, Banach lattices, and operators on these spaces, we suggest [1, 6] (see also [16]). More details on elementary properties of Banach algebras can be found in [9] (see also [8]). Throughout the note, unless stated otherwise, $A$ will stand for an arbitrary ordered Banach algebra with a unit $\mathbf{e} \neq 0$.

\section{The peripheral spectrum of irreducible elements}

An important result in the spectral theory of positive elements is the theorem about the Frobenius normal form. For the case of matrices it means that (see, e.g., [16, p. 31]) an arbitrary non-negative matrix $A$ can be transformed into a block lower-triangular matrix

$$
\left(\begin{array}{cccc}
A_{11} & 0 & \ldots & 0 \\
A_{21} & A_{22} & \ldots & 0 \\
\ldots & \ldots & \ldots & \ldots \\
A_{n 1} & A_{n 2} & \ldots & A_{n n}
\end{array}\right)
$$

using a simultaneous permutation of rows and columns, where the matrices $A_{11}, \ldots, A_{n n}$ are irreducible. We recall that a matrix $D$ is called irreducible [16, p. 19] whenever it can not be transformed to the form $D=\left(\begin{array}{cc}D_{11} & 0 \\ D_{21} & D_{22}\end{array}\right)$ using a simultaneous permutation of rows and columns, where $D_{i i} \neq D$ for $i=1,2$ (the zero $1 \times 1$ matrix is irreducible). Obviously, $\sigma(A)=\bigcup_{i=1}^{n} \sigma\left(A_{i i}\right)$. Therefore, we obtain the dependence of the spectrum of an arbitrary non-negative matrix $A$ on the spectrum of irreducible matrices.

Now we consider the case of an ordered Banach algebra $A$. An element $p \in A$ is called an order idempotent [4] if $0 \leq p \leq \mathbf{e}$ and $p^{2}=p$. Under the partial ordering induced by $A$, the set of all order idempotents $\mathrm{OI}(A)$ of $A$ is a Boolean algebra and its lattice operations satisfy the identities $p \wedge q=p q$ and $p \vee q=p+q-p q$ for all $p, q \in \mathbf{O I}(A)$ (see [4]). For $p \in \mathbf{O I}(A)$ and $a \in A$, we put $p^{\mathrm{d}}=\mathbf{e}-p$ and $a_{p}=p a p$. Obviously, $p^{\mathrm{d}} \in \mathbf{O I}(A)$. A positive element $a \in A$ is said to be order continuous [4] if $p_{\alpha} a \downarrow 0$ and $a p_{\alpha} \downarrow 0$ in $A$ whenever $p_{\alpha} \downarrow 0$ in $\mathrm{OI}(A)$ 1] The collection of all order continuous element of $A$ will be denoted by $A_{\mathrm{n}}$. The algebra $A$ is called order regular if $A_{\mathrm{n}}$ is a subsemi-group of $A$, i.e., $a, b \in A_{\mathrm{n}}$ for every $a, b \in A_{\mathrm{n}}$. Next, an order idempotent $p \in A$ is called a-invariant [4], where $a \in A$, if $p^{\mathrm{d}} a p=0$. A positive element $a \in A$ is said to be irreducible [4] whenever $a$ has no non-trivial (i.e., $p \neq 0$ and $p \neq \mathbf{e}$ ) invariant order idempotents; all other elements of $A$ are called reducible. An element $a \in A^{+}$is said to be irreducible with respect to an order idempotent $p \in \mathrm{OI}(A)$ whenever there exists no $q \in \mathbf{O I}(A)$ such that $0<q<p$ and $(p-q) a q=0$. An element $b \in A$ is called a block [4] of an element $a \in A^{+}$if there exists $a$-invariant order idempotents $p_{1}$ and $p_{2}$ satisfying the relations $p_{2}<p_{1}$ and $b=a_{p_{2} p_{1}^{\mathrm{d}}}$. An element $b$ is called a spectral block of an element $a \in A^{+}$[4] if $b$ is a block of $a$ and $r(b)=r(a)$. An element $a \in A^{+}$is said to have the Frobenius normal form [4]

\footnotetext{
${ }^{1}$ In [5] the notion of order continuity was extended to the case of an arbitrary element $a \in A$, not necessarily positive. However, for our purposes, we can limit oneself to this special situation only.
} 
if there exists $a$-invariant order idempotents $p_{0}, p_{1}, \ldots, p_{n}$, which determine this form, such that $\mathbf{e}=p_{n} \geq p_{n-1} \geq \ldots \geq p_{0}=0$ and if the relation $r\left(a_{p_{i} p_{i-1}^{\mathrm{d}}}\right)=r(a)$ holds for some $i=1, \ldots, n$ then $a_{p_{i} p_{i-1}^{\mathrm{d}}}$ is irreducible with respect to $p_{i} p_{i-1}^{\mathrm{d}}$. In this case, we have the inclusion [4]

$$
\sigma(a) \subseteq \bigcup_{i=1}^{n} \sigma\left(a_{p_{i} p_{i-1}^{\mathrm{d}}}\right)
$$

An order continuous element $a \in A$ is said to be spectrally order continuous [4] if for every spectral block $b$ of $a$ the condition that $r(a)$ is a pole of $R(\cdot, b)$ of order $k$ implies the order continuity of the element $b_{-k}$ (see (1)). The spectral radius $r(a)$ is called a finite-rank pole (abbreviated an $f$-pole) of the resolvent $R(\cdot, a)$ of a positive element $a \in A$ if the inequalities $0 \leq b \leq a$ imply $r(b) \leq r(a)$ and if $r(b)=r(a)$ then $r(a)$ is a pole of $R(\cdot, b)$.

The theorem about the Frobenius normal form has the following form [5]:

Theorem 1. Let an ordered Banach algebra $A$ be Dedekind complete and let an element a of $A$ be spectrally order continuous. Assume that $r(a)>0$ and $r(a)$ is an $f$-pole of $R(\cdot, a)$. Then the element a has the Frobenius normal form.

Example 2. (a) Let us consider the ordered Banach algebra $B(E)$, where $E$ is an arbitrary Banach lattice. If $T \in B(E)$ then, on the one hand, the notion of order continuity can be defined for the operator $T$ on $E$, i.e., [6, p. 46] if a net $x_{\alpha} \stackrel{o}{\longrightarrow} 0$ then $T x_{\alpha} \stackrel{o}{\longrightarrow} 0$. The collection of all order continuous operators on $E$ is denoted by $B_{\mathrm{n}}(E)$. On the other hand, the notion of order continuity can be defined for $T$ as of an element in the ordered Banach algebra $B(E)$ which is defined only for a positive operator. These two notions may differ. Moreover [5], the inclusion $\left(B_{\mathrm{n}}(E)\right)^{+} \subseteq(B(E))_{\mathrm{n}}$ and the converse one do not hold in general. Nevertheless, in the case of a Dedekind complete Banach lattice $E$ these two notions coincide. i.e., we have $\left(B_{\mathrm{n}}(E)\right)^{+}=(B(E))_{\mathrm{n}}$, and, in particular, the algebra $B(E)$ is order regular.

A positive operator $T$ on a Banach lattice $E$ is called (band) irreducible [1, p. 349] if $T$ has no non-trivial invariant bands. Obviously, $\mathrm{OI}(B(E))$ is the collection of all order projections on $E$. If $E$ is Dedekind complete then every band $B$ in $E$ is a projection band and we have the one-to-one correspondence between the set of all bands in $E$ and the set of all order projections on $E$. Therefore, an operator $T$ on a Dedekind complete Banach lattice $E$ is an irreducible operator if and only if $T$ is an irreducible element of the ordered Banach algebra $B(E)$. Next, if $E$ is a Dedekind complete Banach lattice, the Lorenz seminorm $\|x\|_{L}=\inf \left\{\sup \left\|y_{\alpha}\right\|_{E}: 0 \leq y_{\alpha} \uparrow|x|\right\}$ is a norm on $E$ (e.g., the order continuous dual $E_{\mathrm{n}}^{\sim}$ separates points of $E$ or $E$ is an $A M$-space with an order unit), and $T$ is a positive order continuous operator on $E$ with $r(T)>0$ then [4] $r(T)$ is an $f$-pole of the resolvent $R(\cdot, T)$ if and only if $r(T)$ is a finite-rank pole of $R(\cdot, T)$, i.e., $r(T)$ is a pole of $R(\cdot, T)$ and the residue $T_{-1}$ of $R(\cdot, T)$ at $r(T)$ is a finite-rank operator. Various assumptions under which an operator $T$ has the Frobenius normal form in a special case of the algebra $B(E)$ can be found in [4] .

(b) If $E$ is an ordered linear space and an element $x \in E^{+}$then the order ideal $E_{x}$ generated by $x$ is the set [7, p. 103]

$$
E_{x}=\left\{y \in E:-\lambda x \leq y \leq \lambda x \text { for some } \lambda \in \mathbb{R}^{+}\right\}
$$

Under the algebraic operations and the ordering induced be $E, E_{x}$ is a real ordered linear space satisfying $E_{x} \subseteq E^{+}-E^{+}$. If $A$ is an ordered Banach algebra and $b \in A_{\mathrm{n}}$ then $A_{b}^{+} \subseteq A_{\mathrm{n}}$. 
The order ideal $A_{\mathbf{e}}$ is called [5] the center of $A$. As will be shown in Section $6, A_{\mathbf{e}}$ is closed in $A$ and, hence, is a real ordered Banach algebra. Again, if $\mathbf{e} \in A_{\mathrm{n}}$ then $A_{\mathbf{e}}^{+} \subseteq A_{\mathrm{n}}$. However, in general, the inclusion e $\in A_{\mathrm{n}}$ does not hold (see [5]). Nevertheless, in every case, the algebra $A_{\mathbf{e}}$ is order regular. Indeed, let $a \in\left(A_{\mathbf{e}}\right)_{\mathrm{n}}$ and $b \in A_{\mathbf{e}}^{+}$. If $p_{\alpha} \downarrow 0$ in $\mathbf{O I}\left(A_{\mathbf{e}}\right)$ then for some $\lambda \geq 0$, we have $0 \leq p_{\alpha} a b \leq \lambda p_{\alpha} a \downarrow 0$ in $A_{\mathbf{e}}$; analogously, $a b p_{\alpha} \downarrow 0$. Thus, $a b, b a \in\left(A_{\mathbf{e}}\right)_{\mathrm{n}}$.

The author does not know an example of an ordered Banach algebra which is not order regular.

It follows from the theorem about the Frobenius normal form (see Theorem 1 ) and the inclusion (2) that the spectrum of a wide class of positive elements of an ordered Banach algebra $A$ is determined by the spectra of irreducible elements. The next result and Corollary 5 make more precisely this result for the case of the peripheral spectrum.

Lemma 3. Let $B$ be a Banach algebra with a unit $\mathbf{u}$. Let elements $b, p_{0}, p_{1}, \ldots, p_{n} \in B$ with $n \in \mathbb{N}$ satisfy $\left(\mathbf{u}-p_{j}\right) b p_{j}=0, p_{0}=0, p_{n}=\mathbf{u}$, and $p_{i} p_{j}=p_{\min \{i, j\}}$ for all $i, j=0, \ldots, n$. Then $r\left(b_{q_{j}}\right) \leq r(b)$ and

$$
\sigma_{\text {per }}(b)=\bigcup\left\{\sigma_{\text {per }}\left(b_{q_{j}}\right): r\left(b_{q_{j}}\right)=r(b)\right\}
$$

where $q_{j}=p_{j}-p_{j-1}$ and $b_{q_{j}}=q_{j} b q_{j}$ for $j=1, \ldots, n$.

Proof. We mention first that for an arbitrary scalar $\lambda$ belonging to the unbounded connected component $\rho_{\infty}(b)$ of the resolvent set $\rho(b)$ the identity $\left(\mathbf{u}-p_{j}\right) R(\lambda, b) p_{j}=0$ holds for all $j=0, \ldots, n$. Indeed, using an elementary induction, we get $\left(\mathbf{u}-p_{j}\right) b^{n} p_{j}=0$ for all $n \in \mathbb{N}$. Whence, in view of the expansion $R(\lambda, b)=\frac{1}{\lambda}+\frac{1}{\lambda^{2}} b+\ldots$ with $\lambda>r(b)$, we have the identity $\left(\mathbf{u}-p_{j}\right) R(\lambda, b) p_{j}=0$. Taking the unique extension of the analytic function $\left(\mathbf{u}-p_{j}\right) R(\lambda, b) p_{j}$ to $\lambda \in \rho_{\infty}(b)$, we conclude that the last identity holds for all $\lambda \in \rho_{\infty}(b)$; in particular,

$$
\left(\mathbf{u}-p_{j}\right) R(\lambda, b) q_{j}=0
$$

Now let us consider a non-zero scalar $\lambda \in \rho_{\infty}(b)$. The proof will be completed if we will check the invertibility of the element $\lambda-b_{q_{j}}$ for every $j=1, \ldots, n$. To this end, we define the element $z$ via the formula $z=\frac{1}{\lambda}\left(\mathbf{u}-p_{j}\right)+q_{j} R(\lambda, b) q_{j}+\frac{1}{\lambda} p_{j-1}$. Using (4) and the equality $q_{j} b p_{j-1}=0$, we have

$$
\begin{gathered}
\left(\lambda-b_{q_{j}}\right) z=\mathbf{u}-p_{j}+\left(\lambda-b_{q_{j}}\right) q_{j} R(\lambda, b) q_{j}+p_{j-1}=\mathbf{u}-q_{j}+q_{j}\left(\lambda-b_{q_{j}}\right) R(\lambda, b) q_{j}= \\
=\mathbf{u}-q_{j}+q_{j}\left(\lambda-b+b\left(\mathbf{u}-q_{j}\right)\right) R(\lambda, b) q_{j}=\mathbf{u}+q_{j} b\left(\mathbf{u}-q_{j}\right) R(\lambda, b) q_{j}= \\
=\mathbf{u}+q_{j} b\left(\mathbf{u}-p_{j}+p_{j}-q_{j}\right) R(\lambda, b) q_{j}=\mathbf{u}+\left(q_{j} b\left(\mathbf{u}-p_{j}\right)+q_{j} b p_{j-1}\right) R(\lambda, b) q_{j}= \\
=\mathbf{u}+q_{j} b\left(\mathbf{u}-p_{j}\right) R(\lambda, b) q_{j}=\mathbf{u} .
\end{gathered}
$$

Analogously, $z\left(\lambda-b_{q_{j}}\right)=\mathbf{u}$.

As the next example shows, the inclusion (2) cannot make more precisely in general.

Example 4. If $a \in A, q$ is an idempotent of $A$, and $q \neq \mathbf{e}$ then $0 \in \sigma\left(a_{q}\right)$. Thus, the inclusion (2) is proper in general. We shall show that the identity $\sigma(a) \backslash\{0\}=\left(\sigma\left(a_{p^{\mathrm{d}}}\right) \cup \sigma\left(a_{p}\right)\right) \backslash\{0\}$ 
also does not hold, where $p$ is an $a$-invariant order idempotent. To see this, we consider the space $\ell_{\infty}$ of all bounded sequences and define the operator $S$ on $\ell_{\infty}$ via the formula

$$
S x=\left(x_{1}, 0,0,0, x_{3}, 0,0,0, x_{5}, \ldots\right)+\left(0, x_{2}, x_{4}, x_{6}, 0, x_{8}, x_{10}, x_{12}, 0, \ldots\right),
$$

where $x=\left(x_{1}, x_{2}, \ldots\right) \in \ell_{\infty}$. As is easy to see, $S$ is invertible. Put $T=I+S$. Obviously, $1 \notin \sigma(T)$. The band $B=\left\{x \in \ell_{\infty}: x_{2 k}=0\right.$ for all $\left.k \in \mathbb{N}\right\}$ is $T$-invariant and

$$
P_{B} T P_{B} x=\left(x_{1}, 0, x_{3}, 0, x_{5}, 0, x_{7}, 0, x_{9}, \ldots\right)+\left(x_{1}, 0,0,0, x_{3}, 0,0,0, x_{5}, \ldots\right),
$$

where $P_{B}$ is the order projection on $B$. In particular, $1 \in \sigma\left(P_{B} T P_{B}\right)$.

Corollary 5. Let order idempotents $p_{0}, p_{1}, \ldots, p_{n}$ determine the Frobenius normal form of an element $a \in A$. Then

$$
\sigma_{\mathrm{per}}(a)=\bigcup\left\{\sigma_{\mathrm{per}}\left(a_{q_{j}}\right): r\left(a_{q_{j}}\right)=r(a) \text { and } a_{q_{j}} \text { is irreducible with respect to } q_{j}\right\},
$$

where $q_{j}=p_{j} p_{j-1}^{\mathrm{d}}$ for $j=1, \ldots, n$.

Keeping the preceding corollary in mind, we now turn to the study of the peripheral spectrum of irreducible elements. Firstly, let us recall some spectral properties of irreducible elements. It should be mentioned at once that there exists an ordered Banach algebra $A$ such that every positive element of $A$ is irreducible; the latter is equivalent to the identity $\mathbf{O I}(A)=\{0, \mathbf{e}\}$. For example, the ordered Banach algebra $A=A_{0} \otimes \mathbb{C}$ obtained from an ordered Banach algebra $A_{0}$ by adjoining a unit or the algebras $C(K)$ of all continuous functions on $K$ and $B(C(K))$, where $K$ is a connected (Hausdorff) compact. Therefore, in general, one cannot expect any distinctive spectral properties of irreducible elements and, hence, a special class of ordered Banach algebras should be distinguished. We shall say that an ordered Banach algebra $A$ has a disjunctive product [4] if for any $a, b \in A_{\mathrm{n}}$ with $a b=0$ there exists an element $p \in \mathbf{O I}(A)$ satisfying $a p=p^{\mathrm{d}} b=0$. The algebra $B(E)$, where $E$ is a Dedekind complete Banach lattice, has [4] a disjunctive product. Next, an element $a \in A$ is said to be algebraically strictly positive, in symbols $a \gg 0$, whenever $p_{1} a p_{2}>0$ for all $0<p_{1}, p_{2} \in \mathbf{O I}(A)$. The next result holds [4].

Theorem 6. Let $A$ be an ordered algebra with a disjunctive product and with the Boolean algebra $\mathrm{OI}(A)$ Dedekind complete. Assume that a non-zero element a $\in A$ is order continuous, that $r(a)$ is a pole of $R(\cdot, a)$ of order $k$, and that $a_{-k}$ is also order continuous. If the element a is irreducible then the following statements hold:

(a) The spectral radius $r(a)>0$;

(b) The point $r(a)$ is a simple pole of $R(\cdot, a)$;

(c) The residue $a_{-1} \gg 0$ and the resolvent $R(\lambda, a) \gg 0$ for all $\lambda>r(a)$;

(d) If $0 \leq b<a$ and if some element $c \in A_{\mathrm{n}}$ satisfies $0<r(b) c \leq b c$ then $r(b)<r(a)$.

The following lemma and Corollary 8 show that, under additional assumptions on $A$, the condition about the order continuity of the coefficient $a_{-k}$ of the Laurent series expansion of $R(\cdot, a)$ around $r(a)$ in the preceding theorem can be rejected. 
Lemma 7. Let an ordered Banach algebra $A$ be order regular and let $a \in A_{\mathrm{n}}$. Then the resolvent $R(\lambda, a) \in A_{\mathrm{n}}$ for all $\lambda>r(a)$.

Proof. For an arbitrary number $n \in \mathbb{N}$, we define the elements $b_{n}$ and $c_{n}$ as follows

$$
b_{n}=\frac{1}{\lambda} \mathbf{e}+\ldots+\frac{1}{\lambda^{n}} a^{n-1} \text { and } c_{n}=\frac{1}{\lambda^{n+1}} a^{n}+\frac{1}{\lambda^{n+2}} a^{n+1}+\ldots
$$

Obviously, $b_{n}+c_{n}=R(\lambda, a), c_{n} \rightarrow 0$ as $n \rightarrow \infty$, and, since $A$ is order regular, $b_{n} \in A_{\mathrm{n}}$. Let $p_{\alpha} \downarrow 0$ in $\mathbf{O I}(A)$ and let $p_{\alpha} R(\lambda, a) \geq x$ with $x \in A$. Fix an index $\alpha_{0}$. For every $\alpha \geq \alpha_{0}$, the inequality $x \leq p_{\alpha} b_{n}+p_{\alpha_{0}} c_{n}$ holds and, hence, $x-p_{\alpha_{0}} c_{n} \leq p_{\alpha} b_{n} \downarrow_{\alpha \geq \alpha_{0}}$. Therefore, $x \leq p_{\alpha_{0}} c_{n} \rightarrow 0$ as $n \rightarrow \infty$. Finally, $x \leq 0$ or $p_{\alpha} R(\lambda, a) \downarrow 0$. Analogously, $R(\lambda, a) p_{\alpha} \downarrow 0$.

Corollary 8. Let an ordered Banach algebra $A$ be order regular and let $A_{\mathrm{n}}$ be closed in $A$. Let an element $a \in A_{\mathrm{n}}$ such that $r(a)$ is a pole of the resolvent $R(\cdot, a)$ of order $k$. Then $a_{-k} \in A_{\mathrm{n}}$.

Proof. The identity $a_{-k}=\lim _{\lambda \downarrow r(a)}(\lambda-r(a))^{k} R(\lambda, a)$ holds. It only remains to recall the preceding lemma and the closedness of $A_{\mathrm{n}}$.

If $E$ is a Dedekind complete Banach lattice such that the Lorenz seminorm $\|\cdot\|_{L}$ is a norm on $E$ (see Example 2(a)) then [12] the set $(B(E))_{\mathrm{n}}$ is closed in $B(E)$. Consequently, the wide class of ordered Banach algebras of the form $B(E)$ automatically satisfies the assumption about the closedness of $A_{\mathrm{n}}$.

Thus, it follows from Theorem 6 and the preceding corollary that, under the next assumptions on $A$, order continuous irreducible elements have nice spectral properties:

$\left(\mathbf{A}_{1}\right)$ An ordered Banach algebra $A$ is order regular and has a disjunctive product, the Boolean algebra $\mathrm{OI}(A)$ is Dedekind complete, and the set $A_{\mathrm{n}}$ is closed in $A$.

If $E$ is a Dedekind complete Banach lattice and $P$ is a non-zero order continuous projection on $E$ such that $P \gg 0$ as an element of $B(E)$ then [4] $\operatorname{dim} R(P)=1$, where $R(P)$ is the range of the operator $P$. In particular, in view of part (c) of Theorem 6 , "as a rule", the residue $T_{-1}$ of the resolvent $R(\cdot, T)$ of an irreducible operator $T$ at $r(T)$ is a rank-one operator. On the other hand, as is well known, if $Z$ is a Banach space and a projection $Q \in B(Z)$ then $Q$ is a rank-one operator if and only if $Q$ is a minimal idempotent of the algebra $B(Z)$, i.e., $Q B(Z) Q=\mathbb{C} Q$. In the case of an arbitrary ordered Banach algebra $A$ and of an idempotent $b \in A$, the condition $b \gg 0$ does not imply the minimality of the idempotent $b$. For this reason, we must axiomatize this property and make the following assumption:

$\left(\mathbf{A}_{2}\right)$ Every algebraically strictly positive idempotent $b$ of $A$ is minimal.

As usual, through $L_{a}$ and $R_{a}$, we will denote the operators on an algebra $B$ defined by

$$
L_{a} b=a b \text { and } R_{a} b=b a,
$$

where $a, b \in B$, and through $N(S)$, we will denote the null space of the operator $S$ acting between two linear spaces. 
Proposition 9. An ordered Banach algebra A satisfies Axiom $\left(\mathbf{A}_{2}\right)$ if and only iffor an arbitrary idempotent $b \gg 0$ of the following identity holds

$$
\operatorname{dim} N\left(I-L_{b}\right) \cap N\left(I-R_{b}\right)=1 .
$$

In general, the next result is true: If $B$ is a Banach algebra and an idempotent $b \in B$ then $b$ is minimal if and only if (6) holds.

Proof. Let $A$ satisfy Axiom $\left(\mathbf{A}_{2}\right)$ and let $a \in A$ such that $a=b a=a b$. Then $a=b a b=\lambda b$ for some $\lambda \in \mathbb{C}$. For the converse, if $b^{2}=b \gg 0$ then for every $a \in A$ the element $b a b$ belongs to $N\left(I-L_{b}\right) \cap N\left(I-R_{b}\right)$. On the other hand, this space contains $b$ and, hence, $b a b=\mu b$ for some $\mu \in \mathbb{C}$.

For the study of the peripheral spectrum of a positive operator on a Banach lattice, the possibility of the restriction of a problem to the case of operators on the space $C(K)$ of all continuous functions on a compact space $K$ which is simpler for the study, is important. Recall that if $E$ is a Riesz space satisfying Axiom (OS), i.e., [16, p. 54] if the inequalities $0 \leq z_{n} \leq \lambda_{n} z$, where $z_{n}, z \in E$ and $\lambda=\left(\lambda_{1}, \lambda_{2}, \ldots\right) \in \ell_{1}$, imply the existence of $\sup _{n} \sum_{j=1}^{n} z_{j}$, then the order ideal $E_{x}$ generated by $x$ is, under the Minkowski norm $\|\cdot\|_{x}$ defined by

$$
\|y\|_{x}=\inf \left\{\lambda \in \mathbb{R}^{+}:-\lambda x \leq y \leq \lambda x\right\}
$$

with $y \in E_{x}$, an $A M$-space with order unit $x$ [16, p. 102]. Therefore, by the KakutaniBohnenblust-M.-S.Krein theorem [6, p. 201], $E_{x}$ is lattice isometric onto a space $C(K)$ and, moreover, under this isomorphism, $x$ is mapped onto the constant-one function $\mathbb{1}_{K}$. Evidently, every Dedekind complete Riesz space $E$ satisfies Axiom (OS). Now let $E$ be a Dedekind complete Banach lattice. Recall that an operator $T$ on $E$ is said to be regular [6, p. 12] if it can be written as a difference of two positive operators. As is well known, every regular operator is bounded. By the Riesz-Kantorovich theorem [6, p. 14], the space $L_{\mathrm{r}}(E)$ of all regular operators on $E$ is a Dedekind complete Riesz space. Hence, if $T \in L_{\mathrm{r}}(E)$ then the order ideal $(B(E))_{T}$ is lattice isometric onto a space $C(K)$. We axiomatize this property and make the next assumption:

$\left(\mathbf{A}_{3}\right)$ The order ideal $A_{b}$ generated by the non-zero element $b \in A^{+}$is lattice isomorphic onto a space $C(K)$ and, under this isomorphism, $x$ is mapped onto $\mathbb{1}_{K}$.

Every complex Riesz space $E$ is the complexification of the real Riesz space $E_{\mathbb{R}}$ satisfying Axiom (OS) (see [16, Section II.11]). In this case, the algebra $L(E)$ of all operators on $E$ is isomorphic onto the complexification of the algebra $L\left(E_{\mathbb{R}}\right)$ and, in particular, every $T \in L(E)$ has a unique decomposition $T=T_{1}+i T_{2}$, where $T_{j}$ are real maps on $E_{\mathbb{C}}$, i.e., $T_{j}\left(E_{\mathbb{R}}\right) \subseteq E_{\mathbb{R}}$ for $j=1,2$. Defining the set $A_{\mathrm{r}}$ of all regular [5] elements of an ordered Banach algebra $A$ by $A_{\mathrm{r}}=A^{+}-A^{+}$, we axiomatize this property and make the next assumption:

$\left(\mathbf{A}_{4}\right)$ The equality $a+i b=0$ with $a, b \in A_{\mathrm{r}}$ implies $a=b=0$.

We continue our discussion with two auxiliary results.

Lemma 10. Let $B$ be a Banach algebra with a unit, let $b \in B$, and let $m, k \in \mathbb{N}$. If $\lambda_{0} \in \sigma(b)$ and the set $\left\{\lambda_{0}, \lambda_{0} e^{i \frac{2 \pi}{m}}, \ldots, \lambda_{0} e^{i \frac{2 \pi}{m}(m-1)}\right\} \cap \sigma(b)$ consists entirely of poles of $R(\cdot, b)$ of orders which are not greater than $k$, then $\lambda_{0}^{m}$ is a pole of $R\left(\cdot, b^{m}\right)$ of order which is not greater than $k m$. 
Proof. Put $\omega_{j}=e^{i \frac{2 \pi}{m} j}$ with $j=0, \ldots, m-1$. We claim first that $\lambda_{0}^{m}$ is an isolated point of $\sigma\left(b^{m}\right)$. Indeed, if a sequence $\left\{\xi_{n}\right\}$ in $\sigma\left(b^{m}\right)$ satisfies $\xi_{n} \neq \lambda_{0}^{m}$ for all $n$ and $\xi_{n} \rightarrow \lambda_{0}^{m}$ as $n \rightarrow \infty$ then, taking into account the identity $\sigma\left(b^{m}\right)=f(\sigma(b))$ with $f(z)=z^{m}$, we find a sequence $\left\{\mu_{n}\right\}$ in $\sigma(b)$ with the property $\mu_{n}^{m}=\xi_{n}$. Let $\left\{\mu_{n_{r}}\right\}$ be an arbitrary convergent subsequence of $\left\{\mu_{n}\right\}$. If $\mu_{n_{r}} \rightarrow \mu_{0} \in \sigma(b)$ then $\mu_{0}^{m}=\lambda_{0}^{m}$ and, hence, $\mu_{0}=\lambda_{0} \omega_{j}$ for some $j=0, \ldots, m-1$. Therefore, $\mu_{0}$ is an isolated point of $\sigma(b)$. Thus, $\mu_{n_{r}}=\mu_{0}$ for sufficiently large $r$ and for such $r$, we have $\xi_{n_{r}}=\lambda_{0}^{m}$, a contradiction.

For arbitrary numbers $\lambda, z \in \mathbb{C}$ the identity $\lambda^{m}-z^{m}=\prod_{j=0}^{m-1}\left(\lambda-\omega_{j} z\right)$ holds. The latter implies

$$
\lambda^{m}-b^{m}=\prod_{j=0}^{m-1}\left(\lambda-\omega_{j} b\right) .
$$

If $\mathcal{U}_{\lambda_{0}^{m}}$ is a punctured neighbourhood of the point $\lambda_{0}^{m}$ satisfying $\mathcal{U}_{\lambda_{0}^{m}} \subseteq \rho\left(b^{m}\right)$ then the set $\mathcal{V}=\left\{\lambda \in \mathbb{C}: \lambda^{m} \in \mathcal{U}_{\lambda_{0}^{m}}\right\}$ is open and the inclusion $\mathcal{V} \subseteq \rho\left(\omega_{j} b\right)$ holds for all $j=0, \ldots, m-1$. Now, using (8), we obtain

$$
R\left(\lambda^{m}, b^{m}\right)=\prod_{j=0}^{m-1} R\left(\lambda, \omega_{j} b\right)
$$

for all $\lambda \in \mathcal{V}$. Let us consider an arbitrary sequence $\left\{\lambda_{n}\right\}$ in $\mathbb{C}$ satisfying $\lambda_{n} \neq \lambda_{0}^{m}$ for all $n$ and $\lambda_{n} \rightarrow \lambda_{0}^{m}$. It is not difficult to show that there exists a sequence $\left\{\theta_{n}\right\}$ in $\mathbb{C}$ with the properties $\theta_{n} \neq \lambda_{0}^{m}, \theta_{n}^{m}=\lambda_{n}$ for all $n$, and $\theta_{n} \rightarrow \lambda_{0}$ as $n \rightarrow \infty$. Obviously, $\theta_{n} \in \mathcal{V}$ for all sufficiently large $n$. In view of the identity (9), we have

$$
\begin{gathered}
\left(\lambda_{n}-\lambda_{0}^{m}\right)^{k m} R\left(\lambda_{n}, b^{m}\right)=\left(\theta_{n}^{m}-\lambda_{0}^{m}\right)^{k m} R\left(\theta_{n}^{m}, b^{m}\right)=\left(\theta_{n}^{m}-\lambda_{0}^{m}\right)^{k m} \prod_{j=0}^{m-1} R\left(\theta_{n}, \omega_{j} b\right)= \\
=\prod_{j=0}^{m-1}\left(\theta_{n}^{m}-\lambda_{0}^{m}\right)^{k} R\left(\theta_{n}, \omega_{j} b\right)=(-1)^{m+1} \prod_{j=0}^{m-1}\left(\theta_{n}^{m}-\lambda_{0}^{m}\right)^{k} R\left(\theta_{n} \omega_{j}^{-1}, b\right)= \\
=(-1)^{m+1} \prod_{j=0}^{m-1}\left(\theta_{n}^{m}-\lambda_{0}^{m}\right)^{k} R\left(\theta_{n} \omega_{j}, b\right)= \\
=(-1)^{m+1} \prod_{j=0}^{m-1}\left(\sum_{l=0}^{m-1} \theta_{n}^{m-1-l} \lambda_{0}^{l}\right)^{k} \cdot \prod_{j=0}^{m-1}\left(\theta_{n}-\lambda_{0}\right)^{k} R\left(\theta_{n} \omega_{j}, b\right)= \\
=(-1)^{(m+1)(k+1)} \prod_{j=0}^{m-1}\left(\sum_{l=0}^{m-1} \theta_{n}^{m-1-l} \lambda_{0}^{l}\right)^{k} \cdot \prod_{j=0}^{m-1}\left(\theta_{n} \omega_{j}-\lambda_{0} \omega_{j}\right)^{k} R\left(\theta_{n} \omega_{j}, b\right) \rightarrow 0
\end{gathered}
$$

as $n \rightarrow \infty$.

Let $m \in \mathbb{N}$. Consider a nonempty subset $J$ of the set $\{1, \ldots, m\}$. Let $J=\left\{j_{1}, \ldots, j_{r}\right\}$ with $r=1, \ldots, m$. We define the shift $J-1$ of $J$ via the formula $J-1=\left\{j_{1}-1, \ldots, j_{r}-1\right\}$, where in the case of $j_{k}=1$ for some $k=1, \ldots, r$, we put $j_{k}-1=m$. Now, using the elementary induction, the set $J-l$ can be defined easily for every $l \in \mathbb{N}$. Obviously, $J-m=J$. 
Lemma 11. Let $m \in \mathbb{N}$ and let $J$ be a nonempty subset of $\{1, \ldots, m\}$. If $l$ is a minimal natural number satisfying $J-l=J$ then $l$ is a divisor of $m$.

Proof. Evidently, $J-k l=J$ for all $k \in \mathbb{N}$ and $l \leq m$. The representation $m=n l+r$ holds with $n, r \in \mathbb{N}$ and $0 \leq r<l$. Therefore, $J=J-(n l+r)=J-r$. Whence, using the minimality of $l$, we infer $r=0$.

Let be a Banach algebra with a unit. As is well known, if an element $a \in B$ then the identities $\sigma\left(L_{a} ; B(A)\right)=\sigma\left(R_{a} ; B(A)\right)=\sigma(a ; A)$ hold (see (5)). We shall say that a point $\lambda \in \sigma(a)$ is the joint eigenvalue of $a$ whenever there exists a non-zero element $c \in B$ satisfying

$$
a c=c a=\lambda c .
$$

Now let $A$ be an ordered Banach algebra, let $a \in A$, and let $b \in A^{+}$. The joint spectrum of an element $a$ with respect of $b$ is the set $\sigma_{\mathrm{j}}(a ; b ; A)$ of all complex numbers $\lambda$ such that there exists an element $c \in A$ which is not nilpotent, satisfies (10), and has the representation in the form $c=c^{\prime}+i c^{\prime \prime}$, where $c^{\prime}$ and $c^{\prime \prime}$ belong to the order ideal $A_{b}$ (see (3)). Again, when no confusion can occur, we write $\sigma_{\mathrm{j}}(a ; b)$ to denote $\sigma_{\mathrm{j}}(a ; b ; A)$.

Below, through $H_{m}$ with $m \in \mathbb{N}$, we will denote the group of all $m^{\text {th }}$ roots of unity.

Now we are ready to state and to prove the main result of this paper.

Theorem 12. Let an ordered Banach algebra A satisfy Axioms $\left(\mathbf{A}_{1}\right)-\left(\mathbf{A}_{4}\right)$. Let a be a non-zero, order continuous, and irreducible element of $A$ such that $r(a)$ is a pole of $R(\cdot, a)$. Let $m \in \mathbb{N}$, $m>1$. The following statements are equivalent:

(a) $a^{m}$ is reducible;

(b) For some divisor $m^{\prime}$ of $m, m^{\prime}>1$, there exist non-zero order idempotents $p_{1}, \ldots, p_{m^{\prime}}$ of A satisfying $\sum_{j=1}^{m} p_{j}=\mathbf{e}, p_{j^{\prime}} p_{j^{\prime \prime}}=0$ for $j^{\prime} \neq j^{\prime \prime}$, and $p_{j} a=a p_{j+1}$ for all $j=1, \ldots, m^{\prime}$, where for $j=m^{\prime}$, we put $j+1=1$;

(c) For some divisor $m^{\prime \prime}$ of $m, m^{\prime \prime}>1$, and for all $j=0, \ldots, m^{\prime \prime}-1$ the points $r(a) e^{i \frac{2 \pi}{m} j}$ are poles of $R(\cdot, a)$.

If the condition (b) holds then the spectrum $\sigma(a)$ is invariant under the rotation on angle $\frac{2 \pi}{m^{\prime}}$, i.e., $\sigma(a)=\frac{2 \pi}{m^{\prime}} \sigma(a)$.

Moreover, $\sigma_{\mathrm{per}}(a) \cap \sigma_{\mathrm{j}}\left(a ; a_{-1}\right)=r(a) H_{m_{0}}$ for some $m_{0} \in \mathbb{N}$.

Below, we shall say that an arbitrary element $a \in A$ has the cyclic form whenever there exist order idempotents $p_{1}, \ldots, p_{m^{\prime}}$ with $m^{\prime}>1$ satisfying the conditions of part (b). In this case, $p_{1}, \ldots, p_{m^{\prime}}$ is said to determine the cyclic form of $a$.

Proof. (a) $\Longrightarrow$ (b) Taking into account the reducibility of $a^{m}$, we find a non-trivial order idempotent $q_{1}$ of $A$ satisfying $q_{1}^{\mathrm{d}} a^{m} q_{1}=0$. Obviously, $q_{1}^{\mathrm{d}} a a^{m-1} q_{1}=0$. Since the algebra $A$ has a disjunctive product (Axiom $\left(\mathbf{A}_{1}\right)$ ), there exists an order idempotent $q_{2}$ of $A$ such that $q_{1}^{\mathrm{d}} a q_{2}=q_{2}^{\mathrm{d}} a^{m-1} q_{1}=0$. Using the elementary induction, we find $q_{1}, \ldots, q_{m} \in \mathbf{O I}(A)$ satisfying $q_{1}^{\mathrm{d}} a q_{2}=q_{2}^{\mathrm{d}} a q_{3}=\ldots=q_{m}^{\mathrm{d}} a q_{1}=0$. The identity

$$
\prod_{j=1}^{m} q_{j}=0
$$


holds. To see this, keeping the relation

$$
a q_{j}=q_{j-1} a q_{j}
$$

for $j=1, \ldots, m$ (for $j=1$, we put $j-1=m$ ) in mind, we have

$$
\begin{gathered}
\left(\mathbf{e}-\prod_{j=1}^{m} q_{j}\right) a \prod_{j=1}^{m} q_{j}=\left(q_{m}-\prod_{j=1}^{m} q_{j}\right) a \prod_{j=1}^{m} q_{j}= \\
=\left(q_{m} q_{1}-\prod_{j=1}^{m} q_{j}\right) a \prod_{j=1}^{m} q_{j}=\ldots=\left(q_{m} q_{1} \ldots q_{m-1}-\prod_{j=1}^{m} q_{j}\right) a \prod_{j=1}^{m} q_{j}=0 .
\end{gathered}
$$

The irreducibility of $a$ and the relation $q_{1} \neq \mathbf{e}$ imply (11).

For $r \in\{1, \ldots, m\}$, we put $\mathcal{P}_{r}=\{J \subseteq\{1, \ldots, m\}:$ card $J=r\}$. Now we assume the validity of the identity

$$
\prod_{k \in J} q_{k}=0
$$

for all $J \in \mathcal{P}_{r}$, where $r=2, \ldots, m$ and for $r=m$, we obtain (11). Let us show that in this case either there exists the required collection of idempotents or the identity (13) is valid for all $J \in \mathcal{P}_{r-1}$. To this end, let (13) hold for all $J \in \mathcal{P}_{r}, r \geq 2$. Then for all $J_{1}, J_{2} \in \mathcal{P}_{r-1}, J_{1} \neq J_{2}$, we have

$$
\prod_{j \in J_{1}} q_{j} \cdot \prod_{k \in J_{2}} q_{k}=0
$$

We shall say that two subsets $J_{1}, J_{2} \in \mathcal{P}_{r-1}$ is equivalent whenever $J_{1}-l=J_{2}$ for some $l \in \mathbb{N}$ (see the remarks before Lemma 11). As is easy to see, the relation introduced above is an equivalence relation on $\mathcal{P}_{r-1}$ really. Thus, the set $\mathcal{P}_{r-1}$ is the union of (disjoint) equivalence classes $\mathcal{I}_{1}, \ldots, \mathcal{I}_{t}$ with $t \in \mathbb{N}$. Clearly, if $J \in \mathcal{I}_{s}$, where $s \in 1, \ldots, t$, then $J-1 \in \mathcal{I}_{s}$. By Lemma11, $m_{s}=\operatorname{card} \mathcal{I}_{s}$ is a divisor of $m$. If $J \in \mathcal{I}_{s}$ then

$$
\mathcal{I}_{s}=\left\{J, J-1, \ldots, J-\left(m_{s}-1\right)\right\}
$$

Fix $s$. In view of (12), (13), (14), and (15), for an arbitrary set $J_{0} \in \mathcal{I}_{s}$, we have

$$
\begin{gathered}
\left(\mathbf{e}-\sum_{J \in \mathcal{I}_{s}} \prod_{j \in J} q_{j}\right) a \prod_{j \in J_{0}} q_{j}= \\
=\left(\mathbf{e}-\sum_{J \in \mathcal{I}_{s}} \prod_{j \in J} q_{j}\right) \prod_{k \in J_{0}-1} q_{j} \cdot a \prod_{j \in J_{0}} q_{j}=\left(\prod_{k \in J_{0}-1} q_{j}-\prod_{k \in J_{0}-1} q_{j}\right) a \prod_{j \in J_{0}} q_{j}=0 .
\end{gathered}
$$

Since $J_{0}$ is arbitrary, we obtain $\left(\mathbf{e}-\sum_{J \in \mathcal{I}_{s}} \prod_{j \in J} q_{j}\right) a \sum_{J \in \mathcal{I}_{s}} \prod_{j \in J_{0}} q_{j}=0$. Thus, for every $s \in 1, \ldots, t$ either $\sum_{J \in \mathcal{I}_{s}} \prod_{j \in J_{0}} q_{j}=0$ or $\sum_{J \in \mathcal{I}_{s}} \prod_{j \in J_{0}} q_{j}=\mathbf{e}$. Moreover, either the former of these equalities hold for all indexes $s$ or, in view of (14), the second one holds for the unique index $s_{0}$ (if $r=m$ then $s_{0}=t=1$ and $\left.m_{s_{0}}=m\right)$. We consider the second case. Let $\mathcal{I}_{s_{0}}=\left\{J_{1}, \ldots, J_{m_{s_{0}}}\right\}$ and let $J_{j-1}=J_{j}-1$ for all $j=1, \ldots, m_{s_{0}}$. Now we define required order idempotents $p_{1}, \ldots, p_{m_{s_{0}}}$ 
via the formula $p_{j}=\prod_{r \in J_{j}} q_{r}$ for $j=1, \ldots, m_{s_{0}}$. Obviously, $\sum_{j=1}^{m_{s_{0}}} p_{j}=\mathbf{e}$ and $p_{j^{\prime}} p_{j^{\prime \prime}}=0$ for $j^{\prime} \neq j^{\prime \prime}$. Next, for an arbitrary index $j=1, \ldots, m_{s_{0}}$, we have

$$
\begin{gathered}
a p_{j+1}=a \prod_{r \in J_{j}} q_{r}=\sum_{J \in \mathcal{I}_{s_{0}}} \prod_{r \in J} q_{r} \cdot a \cdot \prod_{r \in J_{j+1}} q_{r}= \\
=\left(\sum_{J \in \mathcal{I}_{s_{0}}} \prod_{r \in J} q_{r}\right) \prod_{r \in J_{j+1}-1} q_{r} \cdot a=\left(\sum_{J \in \mathcal{I}_{s_{0}}} \prod_{r \in J} q_{r}\right) \prod_{k \in J_{j}} q_{k} \cdot a=\prod_{r \in J_{j}} q_{r} \cdot a=p_{j} a .
\end{gathered}
$$

The order idempotents $p_{j}$ are non-zero. Indeed, if $p_{j}=0$ for a index $j=1, \ldots, m_{s_{0}}$ then $a p_{j+1}=0$ and, hence, $p_{j+1}^{\mathrm{d}} a p_{j+1}=0$. Thus, $p_{j+1}=0$. Using the elementary induction, we conclude $p_{j}=0$ for all $j$, a contradiction (we have shown that if $p_{1}, \ldots, p_{m^{\prime}}$ satisfies the conditions of part (b) then $p_{j} \neq 0$ for all $j=1, \ldots, m^{\prime}$ and, hence, $p_{j} \neq \mathbf{e}$ for all $j$ ). Now we assume that there exists no such index $s_{0}$. The latter implies the equality $\prod_{k \in J_{j}} q_{k}=0$ for all $J \in \mathcal{I}_{s}$ and all $s$, i.e., the validity of the relation $(13)$ for all $J \in \mathcal{P}_{r-1}$.

Now, using the identity (11), i.e., (13) for $r=m$, and the construction above and taking the finite number of steps, either we will construct the required collection of order idempotents $p_{1}, \ldots, p_{m^{\prime}}$ or we will reduce the problem to the case when the identity (11) holds with $r=1$, i.e., to the case of $q_{1}, \ldots, q_{m}=0$. The latter is impossible as $q_{1} \neq 0$.

(b) $\Longrightarrow$ (a) As was shown above, if $p_{1}, \ldots, p_{m^{\prime}}$ satisfy the condition of part (b) then $p_{j}$ is not-trivial for all $j=1, \ldots, m^{\prime}$. Now, using the elementary induction, we have

$$
p_{j} a^{m^{\prime}}=a p_{j+1} a^{m^{\prime}-1}=a^{2} p_{j+2} a^{m^{\prime}-2}=\ldots=a^{m^{\prime}} p_{j+m^{\prime}}=a^{m^{\prime}} p_{j}
$$

for an arbitrary index $j$, whence

$$
p_{j} a^{m}=p_{j} a^{m^{\prime} \frac{m}{m^{\prime}}}=a^{m^{\prime}} p_{j} a^{\left(m^{\prime}-1\right) \frac{m}{m^{\prime}}}=\ldots=a^{m} p_{j} .
$$

Finally, $p_{j} a^{m} p_{j}^{\mathrm{d}}=0$ and, in particular, $a^{m}$ is reducible.

(b) $\Longrightarrow$ (c) We define the element $d$ via the formula $d=\sum_{j=1}^{m^{\prime}} e^{i \frac{2 \pi}{m^{\prime}} j} p_{j}$, where the order idempotents $p_{1}, \ldots, p_{m^{\prime}}$ satisfy the condition of part (b). As is easy to see, the element $d$ is invertible and $d^{-1}=\sum_{r=1}^{m^{\prime}} e^{-i \frac{2 \pi}{m^{\prime}} j} p_{r}$. We have the equalities

$$
\begin{gathered}
e^{i \frac{2 \pi}{m^{\prime}}} d a d^{-1}=e^{i \frac{2 \pi}{m^{\prime}}}\left(\sum_{j=1}^{m^{\prime}} e^{i \frac{2 \pi}{m^{\prime}} j} p_{j}\right) a\left(\sum_{r=1}^{m^{\prime}} e^{-i \frac{2 \pi}{m^{\prime}} j} p_{r}\right)= \\
=e^{i \frac{2 \pi}{m^{\prime}}} \sum_{j, r=1}^{m^{\prime}} e^{i \frac{2 \pi}{m^{\prime}}(j-r)} p_{j} a p_{r}=e^{i \frac{2 \pi}{m^{\prime}}} \sum_{j=1}^{m^{\prime}} e^{i \frac{2 \pi}{m^{\prime}}(j-(j+1))} a p_{j+1}=a .
\end{gathered}
$$

Therefore, $\sigma(a)=e^{i \frac{2 \pi}{m^{\prime}}} \sigma\left(d a d^{-1}\right)=e^{i \frac{2 \pi}{m^{\prime}}} \sigma(a)$. Consequently, part (b) implies the invariance of the spectrum $\sigma(a)$ under the rotation on angle $\frac{2 \pi}{m^{\prime}}$. In particular, the points $r(a) e^{i \frac{2 \pi}{m^{\prime}} j}$ belong to $\sigma_{\text {per }}(a)$ for $j=0, \ldots, m^{\prime}-1$ as $r(a) \in \sigma(a)$. Let us show that these points are simple 
poles of $R(\cdot, a)$. Using the identity $\lambda-a=e^{i \frac{2 \pi}{m^{\prime}}} d\left(\lambda e^{-i \frac{2 \pi}{m^{\prime}}}-a\right) d^{-1}$ for all $\lambda \in \mathbb{C}$, we obtain $R(\lambda, a)=e^{-i \frac{2 \pi}{m^{\prime}}} d R\left(\lambda e^{-i \frac{2 \pi}{m^{\prime}}}, a\right) d^{-1}$ for all $\lambda \in \rho(a)$. Let $\lambda_{0}$ be a pole of $R(\cdot, a)$ of order $k$. Then $\lambda_{0} e^{i \frac{2 \pi}{m^{\prime}}}$ is an isolated point of $\sigma(a)$ and we have the equalities

$$
\begin{gathered}
\lim _{\lambda \rightarrow \lambda_{0} e^{i \frac{2 \pi}{m^{\prime}}}}\left(\lambda-\lambda_{0} e^{i \frac{2 \pi}{m^{\prime}}}\right)^{k} R(\lambda, a)=\lim _{\lambda \rightarrow \lambda_{0} e^{i \frac{2 \pi}{m^{\prime}}}}\left(\lambda-\lambda_{0} e^{i \frac{2 \pi}{m^{\prime}}}\right)^{k} e^{-i \frac{2 \pi}{m^{\prime}}} d R\left(\lambda e^{-i \frac{2 \pi}{m^{\prime}}}, a\right) d^{-1}= \\
=\lim _{\lambda \rightarrow \lambda_{0} e^{i \frac{2 \pi}{m^{\prime}}}}\left(\lambda e^{-i \frac{2 \pi}{m^{\prime}}}-\lambda_{0}\right)^{k} e^{i \frac{2 \pi}{m^{\prime}} k} e^{-i \frac{2 \pi}{m^{\prime}}} d R\left(\lambda e^{-i \frac{2 \pi}{m^{\prime}}}, a\right) d^{-1}= \\
=\lim _{\mu \rightarrow \lambda_{0}}\left(\mu-\lambda_{0}\right)^{k} e^{i \frac{2 \pi}{m^{\prime}}(k-1)} d R(\mu, a) d^{-1}=0 .
\end{gathered}
$$

Thus, the point $\lambda_{0} e^{i \frac{2 \pi}{m^{\prime}}}$ is a pole of $R(\cdot, a)$ of order which is not greater than $k$. On the other hand, $r(a)$ is a simple pole of $R(\cdot, a)$ and so the points $r(a) e^{i \frac{2 \pi}{m^{\prime}} j}, j=0, \ldots, m^{\prime}-1$, are also simple poles of $R(\cdot, a)$.

(c) $\Longrightarrow$ (a) For $j=0,1, \ldots, m^{\prime \prime}-1$, we put $\omega_{j}=e^{i \frac{2 \pi}{m^{\prime \prime}} j}$. Using the identity [9, p. 22] $\frac{1}{1-z^{m^{\prime \prime}}}=\frac{1}{m^{\prime \prime}} \sum_{j=0}^{m^{\prime \prime}-1} \frac{1}{1-\omega_{j}^{-1} z}$ which is valid for all complex numbers $z \notin\left\{\omega_{0}, \ldots, \omega_{m^{\prime \prime}-1}\right\}$, we have $\frac{1}{\lambda^{m^{\prime \prime}}-z^{m^{\prime \prime}}}=\frac{1}{m^{\prime \prime} \lambda^{m^{\prime \prime}}-1} \sum_{j=0}^{m^{\prime \prime}-1} \frac{\omega_{j}}{\lambda \omega_{j}-z}$ for all $\lambda, z \in \mathbb{C}$ satisfying $\frac{z}{\lambda} \notin\left\{\omega_{0}, \ldots, \omega_{m^{\prime \prime}-1}\right\}$. Therefore, for $\lambda$ from a sufficiently small punctured neighbourhood of $r(a)$, we obtain

$$
m^{\prime \prime} \lambda^{m^{\prime \prime}-1} R\left(\lambda^{m^{\prime \prime}}, a^{m^{\prime \prime}}\right)=\sum_{j=0}^{m^{\prime \prime}-1} \omega_{j} R\left(\lambda \omega_{j}, a\right)
$$

In view of our condition and Lemma 10, $r(a)^{m^{\prime \prime}}$ is a pole of $R\left(\cdot, a^{m^{\prime \prime}}\right)$. Assume that the element $a^{m}$ is irreducible. Then the element $a^{m^{\prime \prime}}$ is also irreducible. Consequently, in view of Axiom $\left(\mathbf{A}_{1}\right), r(a)>0$, the point $r(a)^{m}$ is a simple pole of the resolvent $R\left(\cdot, a^{m^{\prime \prime}}\right)$, and the residue $\left(a^{m^{\prime \prime}}\right)_{-1} \gg 0$. The point $r(a)$ is also a simple pole of the function $\lambda \rightarrow R\left(\lambda^{m^{\prime \prime}}, a^{m^{\prime \prime}}\right)$. Indeed, $r(a)$ is an isolated singular point of this function and for every natural $k \in \mathbb{N}$ the relation

$$
(\lambda-r(a))^{k} R\left(\lambda^{m^{\prime \prime}}, a^{m^{\prime \prime}}\right)=\frac{\left(\lambda^{m^{\prime \prime}}-r(a)^{m^{\prime \prime}}\right)^{k} R\left(\lambda^{m^{\prime \prime}}, a^{m^{\prime \prime}}\right)}{\left(\lambda^{m^{\prime \prime}-1}+\lambda^{m^{\prime \prime}-2} r(a)+\ldots+r(a)^{m^{\prime \prime}-1}\right)^{k}}
$$

holds. Therefore, for every $k>1$, we have

$$
\lim _{\lambda \rightarrow r(a)}(\lambda-r(a))^{k} R\left(\lambda^{m^{\prime \prime}}, a^{m^{\prime \prime}}\right)=0 .
$$

Let $k_{j}$ be the order of the pole of $R(\cdot, a)$ at the point $r(a) \omega_{j}$, where $j=0,1, \ldots, m^{\prime \prime}-1$. We claim equality $k_{j}=1$ (this fact is not assumed in part (c); moreover, we mention that the cone $A^{+}$is not assumed to be normal). Put $l=\max _{0 \leq j \leq m^{\prime \prime}-1} k_{j}$. Proceeding by contradiction, we suppose $l>1$. Therefore, using the identities (16) and (18), we obtain

$$
0=\sum_{j=0}^{m^{\prime \prime}-1} \omega_{j} \lim _{\lambda \rightarrow r(a)}(\lambda-r(a))^{l} R\left(\lambda \omega_{j}, a\right)=
$$




$$
=\sum_{j=0}^{m^{\prime \prime}-1} \lim _{\lambda \rightarrow r(a)}\left(\lambda \omega_{j}-r(a) \omega_{j}\right)^{l} \frac{1}{\omega_{j}^{l-1}} R\left(\lambda \omega_{j}, a\right)=\sum_{j=0}^{m^{\prime \prime}-1} \frac{1}{\omega_{j}^{l-1}} a_{-l, r(a) \omega_{j}}
$$

and so

$$
\sum_{j=0}^{m^{\prime \prime}-1} \frac{1}{\omega_{j}^{l-1}} a_{-l, r(a) \omega_{j}}=0 .
$$

Taking into account the integral representation of the coefficients of the Laurent series expansion of the resolvent $R(\cdot, a)$ around the point $\omega_{j}$ and using the functional calculus, for arbitrary $j_{0}$ satisfying $k_{j_{0}}=l$, we get $a_{-1, r(a) \omega_{j_{0}}} a_{-l, r(a) \omega_{j_{0}}}=a_{-l, r(a) \omega_{j_{0}}}$ and $a_{-1, r(a) \omega_{j_{0}}} a_{-l, r(a) \omega_{j}}=0$ for $j \neq j_{0}$. Now, from the (19), it follows that

$$
0=a_{-1, r(a) \omega_{j_{0}}} \sum_{j=0}^{m^{\prime \prime}-1} \frac{1}{\omega_{j}^{l-1}} a_{-l, r(a) \omega_{j}}=\frac{1}{\omega_{j_{0}}^{l-1}} a_{-l, r(a) \omega_{j_{0}}} .
$$

Therefore, $a_{-l, r(a) \omega_{j_{0}}}=0$, a contradiction. Thus, $l=1$. Next, according to (17),

$$
\lim _{\lambda \rightarrow r(a)}(\lambda-r(a)) R\left(\lambda^{m^{\prime \prime}}, a^{m^{\prime \prime}}\right)=\frac{\left(a^{m^{\prime \prime}}\right)_{-1}}{m^{\prime \prime} r(a)^{m^{\prime \prime}-1}} .
$$

Using the last equality and the identity (17) once more, we obtain

$$
\left(a^{m^{\prime \prime}}\right)_{-1}=\lim _{\lambda \rightarrow r(a)}(\lambda-r(a)) \sum_{j=0}^{m^{\prime \prime}-1} \omega_{j} R\left(\lambda \omega_{j}, a\right)=\sum_{j=0}^{m^{\prime \prime}-1} a_{-1, r(a) \omega_{j}}
$$

and, hence,

$$
\left(a^{m^{\prime \prime}}\right)_{-1} a_{-1, r(a) \omega_{j}}=a_{-1, r(a) \omega_{j}}\left(a^{m^{\prime \prime}}\right)_{-1}=a_{-1, r(a) \omega_{j}} .
$$

In other words, for all $j=0,1, \ldots, m^{\prime \prime}-1$ the idempotents $a_{-1, r(a) \omega_{j}}$ belong to the space $N\left(I-R_{\left(a^{m^{\prime \prime}}\right)_{-1}}\right) \cap N\left(I-L_{\left(a^{m^{\prime \prime}}\right)_{-1}}\right)$. In view of Axiom $\left(\mathbf{A}_{2}\right)$ (see Proposition 9 and, in particular, the identity (6) $)$, this space is one-dimensional. Therefore, taking into account the condition $m^{\prime \prime}>1$, we get $a_{-1, r(a) \omega_{j}}=0$ for all $j$. This contradiction establishes that $a^{m}$ is reducible.

Now we suppose that Axioms $\left(\mathbf{A}_{3}\right)$ and $\left(\mathbf{A}_{4}\right)$ hold and show that for some $m_{0} \in \mathbb{N}$, we have the equality $\sigma_{\text {per }}(a) \cap \sigma_{\mathrm{j}}\left(a ; a_{1}\right)=r(a) H_{m_{0}}$. As is easy to see, in view of Axiom $\left(\mathbf{A}_{4}\right)$, the complexification $\left(A_{a_{-1}}\right)_{\mathbb{C}}$ of the order ideal $A_{a_{-1}}$ generated by $a_{-1}$ is isomorphic onto the complex linear subspace $A_{0}=\left\{b+i c: b, c \in A_{a_{-1}}\right\}$ of $A$. On the other hand, in view of Axiom ( $\left.\mathbf{A}_{3}\right)$, the order ideal $A_{a_{-1}}$ is lattice isomorphic onto a space $C(K)$ and, under this isomorphism, $a_{-1}$ is mapped onto $\mathbb{1}_{K}$. In particular, $A_{a_{-1}}$ is a Riesz space and so the space $(C(K))_{\mathbb{C}}$ can be identified with $A_{0}$, i.e., with the complexification $\left(A_{a_{1}}\right)_{\mathbb{C}}$ of $A_{a_{-1}}$. If for a number $\lambda \geq 0$ and $b \in A_{a_{-1}}$, the inequalities $-\lambda a_{-1} \leq b \leq \lambda a_{-1}$ hold then, taking into account the identity $a a_{-1}=r(a) a_{-1}$, we have $-\lambda r(a) a_{-1} \leq L_{a} b \leq \lambda r(a) a_{-1}$. The latter implies the $L_{a}$-invariance of $A_{a_{-1}}$ and the relation $\left\|L_{a} b\right\|_{a_{-1}} \leq r(a)\|b\|_{a_{-1}}$. Whence $\left\|\widehat{L_{a}}\right\|_{B\left(A_{a_{-1}}\right)}=r(a)$, where the positive operator $\widehat{L_{a}}$ is a restriction of $L_{a}$ to $A_{a_{-1}}$. Thus, $r\left(\widehat{L_{a}}\right)=r(a)$. Since $a_{-1}$ is a minimal idempotent (Axiom $\left(\mathbf{A}_{2}\right)$ ) for arbitrary $b \in A$, we find a scalar $f(b)$ satisfying $a_{-1} b a_{-1}=f(b) a$. The function $f$ on $A_{a_{-1}}$ defined above is linear and positive and satisfies the inequality $|f(b)| \leq\|b\|_{a_{-1}}$ for 
all $b \in A_{a_{-1}}$. In particular, the functional $f$ is bounded. Since $a_{-1} \gg 0, f$ is strictly positive. For arbitrary $a, b \in A_{a_{-1}}$, we have the equalities

$$
f(a b) a_{-1}=a_{-1} a b a_{-1}=r(a) a_{-1} b a_{-1}=r(a) f(b) a_{-1} .
$$

Whence for the adjoint operator ${\widehat{L_{a}}}^{*}$, we get $\left({\widehat{L_{a}}}^{*} f\right)(b)=f(a b)=r(a) f(b)$ or ${\widehat{L_{a}}}^{*} f=r(a) f$.

Fix $\lambda_{0} \in \sigma_{\text {per }}(a) \cap \sigma_{\mathrm{j}}\left(a ; a_{1}\right)$. There exists an element $x_{0}$ of $A$ which is not nilpotent, satisfies the relations $L_{a} x_{0}=R_{a} x_{0}=\lambda_{0} x_{0}$, and has the representation in the form $x_{0}=x_{0}^{\prime}+i x_{0}^{\prime \prime}$, where $x_{0}^{\prime}, x_{0}^{\prime \prime} \in A_{a_{-1}}$. In particular, $x_{0} \in\left(A_{a_{-1}}\right)_{\mathbb{C}}$ and $\widehat{L_{a}} x_{0}=\lambda_{0} x_{0}$. For the element $x_{0}$ the modulus $\left|x_{0}\right|$ exists, belongs to $A_{a_{-1}}$, and is given by $\left|x_{0}\right|=\sup _{\varphi \in[0,2 \pi]}\left|(\cos \varphi) x_{0}^{\prime}+(\sin \varphi) x_{0}^{\prime}\right|$. We can assume $\left\|\left|x_{0}\right|\right\|_{a_{-1}}=1$. For the element $a x_{0}=a x_{0}^{\prime}+i a x_{0}^{\prime \prime} \in\left(A_{a_{-1}}\right)_{\mathbb{C}}$ and for the modulus of this element in $\left(A_{a_{-1}}\right)$ , we have

$$
\left|a x_{0}\right|=\sup _{\varphi \in[0,2 \pi]}\left|(\cos \varphi) a x_{0}^{\prime}+(\sin \varphi) a x_{0}^{\prime}\right| \leq a \sup _{\varphi \in[0,2 \pi]}\left|(\cos \varphi) x_{0}^{\prime}+(\sin \varphi) x_{0}^{\prime}\right|=a\left|x_{0}\right| .
$$

Whence $r(a)\left|x_{0}\right|=\left|\lambda_{0} x_{0}\right|=\left|a x_{0}\right| \leq a\left|x_{0}\right|$ or $0 \leq(a-r(a))\left|x_{0}\right|$. On the other hand, $0=a_{-1}(a-r(a))\left|x_{0}\right|$ and $a_{-1} \gg 0$. Thus, $r(a)\left|x_{0}\right|=a\left|x_{0}\right|$; analogously, $r(a)\left|x_{0}\right|=\left|x_{0}\right| a$. Therefore, for $\lambda>r(a)$, we have

$$
R(\lambda, a)\left|x_{0}\right|=\frac{1}{\lambda}\left|x_{0}\right|+\frac{1}{\lambda^{2}} r(a)\left|x_{0}\right|+\ldots=\frac{\left|x_{0}\right|}{\lambda}\left(1+\frac{r(a)}{\lambda}+\left(\frac{r(a)}{\lambda}\right)^{2}+\ldots\right)=\frac{\left|x_{0}\right|}{\lambda_{0}-r(a)}
$$

and, hence, $a_{-1}\left|x_{0}\right|=\left|x_{0}\right|$; analogously, $\left|x_{0}\right| a_{-1}=\left|x_{0}\right|$. Consequently, we have the equality $\left|x_{0}\right|=a_{-1}\left|x_{0}\right|=a_{-1}\left|x_{0}\right| a_{-1}=\mu a_{-1}$ for some $\mu \in \mathbb{R}$. Since $\left\|\left|x_{0}\right|\right\|_{a_{-1}}=1$, we infer $\mu=1$ and so $\left|x_{0}\right|=a_{-1}$. There exists (see [14, Lemma 5.1(I)]) an operator $S$ on $\left(A_{a_{-1}}\right)_{\mathbb{C}}$ depending upon $x_{0}$ and satisfying the identity $\widehat{L_{a}}=r(a) \lambda_{0}^{-1} S^{-1} \widehat{L_{a}} S$; analogously, $\widehat{R_{a}}=r(a) \lambda_{0}^{-1} S^{-1} \widehat{R_{a}} S$ for the same operator $S$, where $\widehat{R_{a}}$ is a restriction of $R_{a}$ to $A_{a_{-1}}$. Now, if $\lambda^{\prime} \in \sigma_{\text {per }}(a) \cap \sigma_{\mathrm{j}}\left(a ; a_{1}\right)$ and $L_{a} x=R_{a} x=\lambda^{\prime} x$ for $x \in\left(A_{a_{-1}}\right)_{\mathbb{C}}$ then $\widehat{L_{a}} S x=\frac{\lambda_{0} \lambda^{\prime}}{r(a)} S x$ and $\widehat{R_{a}} S x=\frac{\lambda_{0} \lambda^{\prime}}{r(a)} S x$. Whence $\frac{\lambda_{0} \lambda^{\prime}}{r(a)}$ belongs to the set $\sigma_{\text {per }}(a) \cap \sigma_{\mathrm{j}}\left(a ; a_{1}\right)$. Finally, since $\lambda_{0}$ and $\lambda^{\prime}$ are arbitrary, the set $\frac{1}{r(a)} \sigma_{\text {per }}(a) \cap \sigma_{\mathrm{j}}\left(a ; a_{1}\right)$ is the group of all $m_{0}^{\text {th }}$ roots of unity for some $m_{0} \in \mathbb{N}$. We used Axiom $\left(\mathbf{A}_{4}\right)$ where $b$ is an algebraically strictly positive idempotent only. We didn't use the assumption that $x_{0}$ is not nilpotent (this assumption from the definition of $\sigma_{\mathrm{j}}(a ; b)$ will be needed for the validity of Lemma 15 and Corollary 16 ).

The proof of the theorem is now complete.

In the proof of the preceding theorem, using a number $m$ of part (a), a number $m^{\prime}$ satisfying the conditions of part (b) was found. A number $m^{\prime}$ is not uniquely determined. Indeed, for the cyclic matrix $\left(\begin{array}{llll}0 & 1 & 0 & 0 \\ 0 & 0 & 1 & 0 \\ 0 & 0 & 0 & 1 \\ 1 & 0 & 0 & 0\end{array}\right)$ two situations $m^{\prime}=2$ and $m^{\prime}=4$ are possible (then the required order idempotents of part (b) correspond, in the former, to the coordinates $\{1,3\}$ and $\{2,4\}$ and, in the second case, to $\{1\}, \ldots,\{4\})$. For the case of the analogous cyclic $6 \times 6$ matrix three situations $m^{\prime}=2(\{1,3,5\}$ and $\{2,4,6\}), m^{\prime}=3(\{1,4\},\{2,5\}$, and $\{3,6\})$, and $m^{\prime}=6$ $(\{1\}, \ldots,\{6\})$ are possible.

As is easy to see from the proof of Theorem 12 , if $p_{1}, \ldots, p_{m^{\prime}}$ satisfying the conditions of part (b) exist then part (c) holds with $m^{\prime \prime}=m^{\prime}$. In is not known if the converse is valid. I.e., does part (b) hold with $m^{\prime \prime}=m^{\prime}$ if part (c) holds? In other words, the author does not know 
the direct proof of the implication $(\mathbf{c}) \Longrightarrow(\mathbf{b})$ of the preceding theorem. Nevertheless, in the following particular case such a proof is possible. Let the condition of part (c) of Theorem 12 hold and let the number $m^{\prime \prime}$ have the representation in the form of the product $m^{\prime \prime}=m_{1} \ldots m_{n}$, where $n \in \mathbb{N}, m_{k}$ are prime numbers for all $k=1, \ldots, n$, and $m_{k^{\prime}} \neq m_{k^{\prime \prime}}$ for all $k^{\prime} \neq k^{\prime \prime}$. Obviously, for arbitrary $k=1, \ldots, n$ and all $j=0,1, \ldots, m_{k}-1$ the points $r(a) e^{i \frac{2 \pi}{m_{k}} j}$ are poles of $R(\cdot, a)$ and so (see the proof of the implication (c) $\Longrightarrow(\mathbf{a})) a^{m_{k}}$ is reducible. In view of the validity of the implication (a) $\Longrightarrow$ (a) and since $m_{k}$ is a prime number, there exists non-zero order idempotents $p_{m_{k}, 1}, \ldots, p_{m_{k}, m_{k}}$ determining the cyclic form of the element $a$. For an arbitrary $n$ tuple $\left(j_{1}, \ldots, j_{n}\right)$, where $j_{k}=1, \ldots, m_{k}$ for all $k=1, \ldots, n$, we put $p_{j_{1}, \ldots, j_{n}}=p_{m_{1}, j_{1}} \ldots p_{m_{n}, j_{n}}$. If $\left(j_{1}^{\prime}, \ldots, j_{n}^{\prime}\right) \neq\left(j_{1}^{\prime \prime}, \ldots, j_{n}^{\prime \prime}\right)$ then $p_{j_{1}^{\prime}, \ldots, j_{n}^{\prime}} \perp p_{j_{1}^{\prime \prime}, \ldots, j_{n}^{\prime \prime}}$ and, hence, the number of order idempotents $p_{j_{1}, \ldots, j_{n}}$ is equal to $m^{\prime \prime}$. Next, $\sum_{j_{1}=1}^{m_{1}} \ldots \sum_{j_{n}=1}^{m_{n}} p_{j_{1}, \ldots, j_{n}}=\mathbf{e}, p_{j_{1}, \ldots, j_{n}} a=a p_{j_{1}-1, \ldots, j_{n}-1}$, and $p_{j_{1}-m^{\prime \prime}, \ldots, j_{n}-m^{\prime \prime}}=p_{j_{1}, \ldots, j_{n}}$. If for some $r \in \mathbb{N}$ the equality $p_{j_{1}-r, \ldots, j_{n}-r}=p_{j_{1}, \ldots, j_{n}}$ is valid then for all $k=1, \ldots, n$ the number $m_{k}$ is a divisor of $r$. Therefore, $r=m_{1} \ldots m_{k} l=m^{\prime \prime} l$ with $l \in \mathbb{N}$. Finally, the collection $\left\{p_{j_{1}, \ldots, j_{n}}\right\}$ determines the cyclic form of $a$.

The next result makes more precisely correlations between the values $m, m^{\prime}$, and $m^{\prime \prime}$.

Corollary 13. Under the assumptions of Theorem 12 the following equalities hold

$$
\begin{gathered}
\min \left\{m \in \mathbb{N}: a^{m} \text { is reducible }\right\}= \\
=\min \left\{m \in \mathbb{N}: \text { there exist } p_{1}, \ldots, p_{m} \text { in } \mathbf{O I}(A) \text { determining the cyclic form of } a\right\} \\
=\min \left\{m \in \mathbb{N}: r(a) e^{i \frac{2 \pi}{m} j} \text { is a pole of } R(\cdot, a) \text { for } j=0,1, \ldots, m-1\right\} ;
\end{gathered}
$$

if $a^{m}$ is irreducible for all $m$ or such $p_{1}, \ldots, p_{m}$ does not exist then we suppose that the respective minimum is equal to one.

We continue with the following two auxiliary results.

Lemma 14. Let $c \in A^{+}$, let $p_{0}, p_{1}, \ldots, p_{n}$, where $n \in \mathbb{N}$, be a collection of c-invariant order idempotents satisfying the relations $\mathbf{e}=p_{n} \geq \ldots \geq p_{1} \geq p_{0}$, and let $q_{j}=p_{j} p_{j-1}^{\mathrm{d}}$ for all $j=1, \ldots, n$. If for every $j$ the elements $c_{q_{j}}$ are nilpotent then the element $c$ is also nilpotent.

Proof. For $n=1$ the assertion is obvious. Let us assume $n>1$. We consider first the case of $c_{q_{j}}=0$ for all $j$ and will show the identity $c^{n}=0$. To this end, using the induction on $k=1, \ldots, n$, we shall prove the validity of the equality $q_{s} c^{k} q_{j}=0$ for all $j=1, \ldots, n$ and $s=\max \{1, j-k+1\}, \ldots, n$ which, for $k=n$, implies $q_{s} c^{n} q_{j}=0$ for all $s$ and $j$. In view of the relation $\sum_{j=1}^{n} q_{j}=\mathbf{e}$, we obtain $c^{n}=0$. If $j<s$ then $0 \leq q_{s} c q_{j} \leq p_{s-1}^{\mathrm{d}} c p_{j} \leq p_{j-1}^{\mathrm{d}} c p_{j}=0$. Taking into account our condition, we have the equality $q_{s} c q_{j}=0$ for $j \leq s$. Therefore, for $k=1$ our induction hypothesis is true and the identity $c=\sum_{s=1}^{n-1} \sum_{j=s+1}^{n} q_{s} c q_{j}$ holds. Assume the validity of our assertion for some $k<m$. Since $n-k+1 \geq \max \{1, j-k+1\}$ for all $j$, we obtain $q_{s} c^{k} q_{j}=0$ for $s \geq n-k+1$ and this equality also holds for $j<s+k$. Therefore, $c^{k}=\sum_{s=1}^{n-k} \sum_{j=s+k}^{n} q_{s} c^{k} q_{j}$. Evidently, $q_{m} c^{k+1} q_{j}=0$ for all $j$ and $q_{s} q_{j}=0$ for $s \neq j$. Now, for 
arbitrary indexes $l$ and $t$ with $l \leq m-1$, we have

$$
q_{l} c^{k+1} q_{t}=q_{l} c c^{k} q_{t}=\sum_{j=l+1}^{n} q_{l} c q_{j} \cdot \sum_{s=1}^{n-k} \sum_{j=s+k}^{n} q_{s} c^{k} q_{j} q_{t}=\sum_{j=l+1}^{n} q_{l} c q_{j} \cdot \sum_{s=1}^{t-k} q_{s} c^{k} q_{t} .
$$

Consequently, if $t-k \leq l$ then $q_{l} c^{k+1} q_{t}=0$, as desired.

In a general case, we mention first the validity of the equality $\left(c^{k}\right)_{q_{j}}=\left(c_{q_{j}}\right)^{k}$ for all $k \in \mathbb{N}$ and $j=1, \ldots, n$. Indeed, the case of $k=1$ is obvious. If the required equality is true for some $k$ then

$$
\left(c_{q}\right)^{k+1}=\left(c_{q}\right)^{k} c_{q}=\left(c^{k}\right)_{q} c_{q}=q c^{k} q c q=q c^{k+1} q-q c^{k} q^{\mathrm{d}} c q=\left(c^{k+1}\right)_{q} .
$$

Now we choose $k_{j} \in \mathbb{N}$ satisfying $c_{q_{j}}^{k}=0$ for all $j=1, \ldots, n$. Putting $k=\max _{1 \leq j \leq n} k_{j}$, we have $\left(c^{k}\right)_{q_{j}}=\left(c_{q_{j}}\right)^{k}=0$. As was showed above, $c^{k n}=0$.

For a non-zero element $q \in \mathbf{O I}(A)$, we put $A(q)=\{a \in A: q a q=a\}$. Under the linear operations, the multiplication, the norm, and the order induced by $A$, the linear space $A(q)$ is an ordered Banach algebra with unit $q$ being a closed subalgebra of $A$. We mention at once the following properties of $A(q)$ [4]: (a) For the order interval $[0, q]$ in $A$ the identity $\mathbf{O I}(A(q))=\mathbf{O I}(A) \cap[0, q]$ is valid; (b) An element $z \in A(q)$ is irreducible with respect $q$ if and only if $z$ is irreducible in $A(q)$; (c) The identity $(A(q))_{\mathrm{n}}=A_{\mathrm{n}} \cap A(q)$ holds; (d) If $A$ has a disjunctive product then $A(q)$ also has a disjunctive product. From this remarks, it follows that if $A$ satisfies Axiom ( $\mathbf{A}_{j}$ ) for some $j=1,3,4$ then $A(q)$ also satisfies this axiom.

Lemma 15. Let $A$ satisfy Axiom $\left(\mathbf{A}_{1}\right)$. Let an element $a \in A_{\mathrm{n}}$ such that $r(a)>0$ is a pole of $R(\cdot, a)$ and the residue $a_{-1}$ possesses a modulus $\left|a_{-1}\right|$. Let the collection of order idempotents $p_{0}, p_{1}, \ldots, p_{n}$ determine the Frobenius normal form of the element $a$. Then the inclusions

$$
\begin{gathered}
\sigma_{\mathrm{per}}(a ; A) \cap \sigma_{\mathrm{j}}\left(a ;\left|a_{-1}\right| ; A\right) \subseteq \\
\subseteq \bigcup\left\{\sigma_{\mathrm{per}}\left(a_{q_{j}} ; A\left(q_{j}\right)\right) \cap \sigma_{\mathrm{j}}\left(a_{q_{j}} ;\left(a_{q_{j}}\right)_{-1} ; A\left(q_{j}\right)\right): r\left(a_{q_{j}}\right)=r(a)\right\} \subseteq \sigma_{\mathrm{per}}(a ; A)
\end{gathered}
$$

hold with $q_{j}=p_{j} p_{j-1}^{\mathrm{d}}$ for $j=1, \ldots, n$.

Proof. We denote the middle part of (20) by $\mathcal{S}$. By Corollary 5, to check the left inclusion in (20), it suffices to establish the relation

$$
\bigcup\left\{\sigma_{\mathrm{per}}\left(a_{q_{j}} ; A\right) \cap \sigma_{\mathrm{j}}\left(a ;\left|a_{-1}\right| ; A\right): r\left(a_{q_{j}}\right)=r(a)\right\} \subseteq \mathcal{S}
$$

To this end, let $\lambda \in \sigma_{\mathrm{j}}\left(a ;\left|a_{-1}\right| ; A\right)$ and let $|\lambda|=r(a)$. Then there exists an element $c \in A$ which is not nilpotent, satisfies $a c=c a=\lambda c$, and has a representation in the form $c=c^{\prime}+i c^{\prime \prime}$ with $c^{\prime}, c^{\prime \prime} \in A_{\left|a_{-1}\right|}$. For arbitrary $j=0,1, \ldots, n$, the order idempotent $p_{j}$ is $\left|a_{-1}\right|$-invariant and, hence, is $c$-invariant. Therefore, for $j=1, \ldots, n$, we have

$$
q_{j}^{\mathrm{d}} c q_{j}=\left(p_{j}^{\mathrm{d}}+p_{j-1}\right) c p_{j} p_{j-1}^{\mathrm{d}}=p_{j-1} c q_{j} ;
$$

analogously, $q_{j} a q_{j}^{\mathrm{d}}=q_{j} c p_{j}^{\mathrm{d}}$. Thus, $q_{j} a q_{j}^{\mathrm{d}} c q_{j}=0$. Consequently,

$$
\lambda c_{q_{j}}=q_{j} a c q_{j}=q_{j} a q_{j} c q_{j}+q_{j} a q_{j}^{\mathrm{d}} c q_{j}=a_{q_{j}} c_{q_{j}}
$$


analogously, $\lambda c_{q_{j}}=c_{q_{j}} a_{q_{j}}$. In view of Lemma 14, there exists an index $j_{0}$ such that $c_{q_{j_{0}}}$ is not nilpotent. In particular, $c_{q_{j_{0}}} \neq 0$ and, hence, $\lambda \in \sigma\left(a_{q_{j_{0}}}\right)$. On the other hand, Lemma 3 yields $r\left(a_{q_{j_{0}}}\right) \leq r(a)$. Therefore, $r\left(a_{q_{j_{0}}}\right)=r(a), a_{q_{j_{0}}}$ is irreducible with respect $q_{j_{0}}$, and $\lambda \in \sigma_{\text {per }}\left(a_{q_{j_{0}}} ; A\left(q_{j_{0}}\right)\right)$. Then [4] $r(a)$ is a simple pole of $R\left(\cdot, a_{q_{j_{0}}}\right)$ and for $\lambda$ from a sufficient small punctured neighbourhood of $r(a)$ the identity

$$
R\left(\lambda, a_{q_{j_{0}}}\right)=\frac{1}{\lambda}\left(p_{j_{0}}^{\mathrm{d}}+p_{j_{0}-1}\right)+q_{j_{0}} R(\lambda, a) q_{j_{0}}
$$

holds. Thus, $0 \leq\left(a_{q_{j_{0}}}\right)_{-1}=\left(a_{-1}\right)_{q_{j_{0}}}$. The last equality implies $\left(c^{\prime}\right)_{q_{j_{0}}},\left(c^{\prime \prime}\right)_{q_{j_{0}}} \in A_{\left(a_{q_{j_{0}}}\right)_{-1}}$ and, hence, $\lambda \in \sigma_{\mathrm{j}}\left(a_{q_{j_{0}}} ;\left(a_{q_{j_{0}}}\right)_{-1} ; A\left(q_{j_{0}}\right)\right)$.

To check the right inclusion in (20), mention the next inclusion $\sigma_{\text {per }}\left(a_{q} ; A(q)\right) \subseteq \sigma_{\text {per }}\left(a_{q} ; A\right)$ for arbitrary $q \in \mathbf{O I}(A)$. In fact, if $\left(\lambda-a_{q}\right) b=b\left(\lambda-a_{q}\right)=\mathbf{e}$ for an element $b \in A$ then $\left(\lambda-a_{q}\right) b_{q}=b_{q}\left(\lambda-a_{q}\right)=q$. Now it only remains to recall Lemma 3 .

Theorem 12 and the preceding lemma imply the next consequence which characterizes the peripheral spectrum of an arbitrary positive element of $A$.

Corollary 16. Let the assumptions of Lemma 15 satisfy, let A satisfy Axioms $\left(\mathbf{A}_{3}\right)$ and $\left(\mathbf{A}_{4}\right)$, and let the algebra $A(q)$ satisfy Axiom $\left(\mathbf{A}_{2}\right)$ for every non-zero $q \in \mathrm{OI}(A)$. Then the inclusions

$$
\sigma_{\mathrm{per}}(a ; A) \cap \sigma_{\mathrm{j}}\left(a ;\left|a_{-1}\right| ; A\right) \subseteq r(a) \bigcup_{s=1}^{n} H_{m_{s}} \subseteq \sigma_{\mathrm{per}}(a ; A)
$$

hold with some $m_{1}, \ldots, m_{n} \in \mathbb{N}$.

We close this section with the next assertion about elements having the cyclic form.

Proposition 17. Let an element $a \in A$ have the cyclic form. Suppose that there exists at least one pole of the resolvent $R(\cdot, a)$ among points of $\sigma_{\mathrm{per}}(a)$. Then the decomposition $a^{m}=\sum_{j=1}^{m} b_{j}$ holds, where $1<m \in \mathbb{N}, b_{j^{\prime}} b_{j^{\prime \prime}}=0$ for $j^{\prime} \neq j^{\prime \prime}$, and $r\left(b_{j}\right)=r(a)^{m}$ for all $j=1, \ldots, m$. If, in addition, $a \in A^{+}$then there exists such a decomposition that $b_{j^{\prime}} \wedge b_{j^{\prime \prime}}=0$ for $j^{\prime} \neq j^{\prime \prime}$.

Proof. Using our condition, we find order idempotents $p_{1}, \ldots, p_{m}$ of $A$, where $m>1$, satisfying $p_{j} a=a p_{j+1}$ for $j=1, \ldots, m$. Evidently, $p_{j} a^{m}=a p_{j+1} a^{m-1}=\ldots=a^{m} p_{j}$, whence $p_{j} a^{m} p_{j}=a^{m} p_{j}=p_{j} a^{m}$ for all $j$ and $p_{j^{\prime}} a^{m} p_{j^{\prime \prime}}=0$ for $j^{\prime} \neq j^{\prime \prime}$. Therefore, putting $b_{j}=p_{j} a^{m} p_{j}$, we obtain $a^{m}=\sum_{j=1}^{m} b_{j}$. Fix an index $j$. Let $\lambda_{0}$ be a pole of $R(\cdot, a)$ and let $\left|\lambda_{0}\right|=r(a)$. Since the equality $p_{j} R(\lambda, a)=0$ is impossible $\lambda$ for sufficiently close to $\lambda_{0}$, $\lambda \neq \lambda_{0}$, there exists a number $s \in \mathbb{N}$ satisfying $p_{j} a_{\lambda_{0}, s} \neq 0$ and $p_{j} a_{\lambda_{0}, t}=0$ for $t<s$. Keeping the identity $a a_{\lambda_{0}, s}=r(a) a_{\lambda_{0}, s}+a_{\lambda_{0}, s-1}$ in mind and using the elementary induction, it is not difficult to check the validity of the relation $a^{n} a_{\lambda_{0}, s}=\sum_{t=0}^{n} \lambda_{0}^{t} C_{n}^{t} a_{\lambda_{0}, s-(n-t)}$ for all $n \in \mathbb{N}$, where $C_{n}^{t}$ are binomial coefficients. The latter implies the equality $p_{j} a^{m} a_{\lambda_{0}, s}=\lambda_{0}^{m} p_{j} a_{\lambda_{0}, s}$ or $b_{j} a_{\lambda_{0}, s}=\lambda_{0}^{m} a_{\lambda_{0}, s}$. Thus, $\lambda_{0}^{m} \in \sigma\left(b_{j}\right)$. Taking into account the inequality $r\left(b_{j}\right) \leq r(a)^{m}$, we have $r\left(b_{j}\right)=r(a)^{m}$. In view of the definition of $b_{j}$, the last assertion is clear. 


\section{The Lotz-Schaefer axiom}

In the spectral theory of positive operators the next Lotz-Schaefer theorem is one of the most significant results (see [16, pp. 351-352]). In some situations, e.g., in the case of irreducible operators (see [2]), this theorem allows the study of points of the peripheral spectrum of an operator to reduce to the case of poles only.

Theorem 18. Let $T$ be a positive operator on a Banach lattice $E$ and let $r(T)$ be a finite-rank pole of $R(\cdot, T)$. Then the peripheral spectrum $\sigma_{\text {per }}(T)$ of $T$ consists entirely of poles of $R(\cdot, T)$.

As was mentioned above (see Axiom $\left(\mathbf{A}_{2}\right)$ and the remarks before this axiom), algebraically strictly positive projections in an ordered Banach algebra $A$ can be considered as a generalization of rank-one operators. Moreover, the residue $a_{-1}$ of the resolvent $R(\cdot, a)$ of the irreducible element $a$ at the point $r(a)$ satisfies this condition (see Theorem 6(c)). An element $b \in A$ is said to be relatively algebraically strictly positive whenever there exists a non-zero order idempotent $q$ of $A$ such that $b \in A(q)$ and $q_{1} a q_{2}>0$ for all $0<q_{1}, q_{2} \in \mathbf{O I}(A) \cap[0, q]$; in this case, we write $b \gg_{q} 0$. Obviously, $b \gg 0$ if and only if $b \gg_{\mathbf{e}} 0$. As is easy to see, if $P$ is a non-zero order continuous projection on a Dedekind complete Banach lattice $E$ being relatively algebraically strictly positive element in the ordered Banach algebra $B(E)$ then $\operatorname{dim} R(P)=1$. On the other hand, the collection $F(E)$ of finite-rank operators on $E$ is an algebraic ideal of $B(E)$ and if a non-zero operator $T \in F(E)$ then the algebraic ideal generated by $T$ in $B(E)$ coincides with $F(E)$. Keeping these remarks in mind, we define the set $\mathcal{F}(A)$ of finite-rank elements of an ordered Banach algebras $A$ as the (two-sided) algebraic ideal generated by the set of all relatively algebraically strictly positive order continuous projectors of $A$; if such projectors do not exist, we put $\mathcal{F}(A)=\emptyset$. If $a \in A(q)$ is a relatively algebraically strictly positive element of $A(q)$ then $a$ is such an element of $A$, and, hence, $\mathcal{F}(A(q)) \subseteq \mathcal{F}(A)$. Now, axiomatizing the respective theorem, we can introduce the Lotz-Schaefer axiom in the following manner:

( $\left.\mathbf{A}_{\mathrm{LS}}\right)$ If $a$ is a positive element of $A, r(a)$ is a pole of $R(\cdot, a)$, and the residue $a_{-1}$ is a finite-rank element then the peripheral spectrum $\sigma_{\text {per }}(a)$ of $a$ consists entirely of poles of $R(\cdot, a)$.

If $E$ is a Dedekind complete Banach lattice admitting a weak order unit $x>0$ and a strictly positive order continuous functional $f$ then the projection $\frac{1}{f(x)} f \otimes x \gg 0$ in the ordered Banach algebra $B(E)$ and, hence, $\mathcal{F}(B(E))=F(E)$. Therefore, the Lotz-Schaefer theorem implies the validity of Axiom $\left(\mathbf{A}_{\mathrm{LS}}\right)$ for a wide class of ordered Banach algebras of the form $B(E)$. If we want the validity of a similar axiom for a wider class of algebras of the form $B(E)$ then we must introduce the next weaker axiom:

$\left(\mathbf{A}_{\mathrm{LS}}^{\prime}\right)$ If $a$ is a positive element of $A, r(a)$ is a pole of $R(\cdot, a)$, the residue $a_{-1}$ is an order continuous element, and $a_{-1} \gg 0$ then the peripheral spectrum $\sigma_{\text {per }}(a)$ of $a$ consists entirely of poles of $R(\cdot, a)$.

Now if $E$ is an arbitrary Dedekind complete Banach lattice then the ordered Banach algebra $B(E)$ satisfies Axiom $\left(\mathbf{A}_{\mathrm{LS}}^{\prime}\right)$. Moreover, the last two conditions of this axiom automatically hold when the element $a$ is irreducible (see Theorem 6(c)). However, as the results below show (see, e.g., Theorem 23), in the case of an arbitrary positive elements Axiom $\left(\mathbf{A}_{\mathrm{LS}}\right)$ is more useful than Axiom $\left(\mathbf{A}_{\mathrm{LS}}^{\prime}\right)$. 
Nevertheless, there are other cases when an algebra $A$ need not satisfy Axiom $\left(\mathbf{A}_{\mathrm{LS}}\right)$. If $\mathrm{OI}(A)=\{0, \mathbf{e}\}$ then e $\gg 0$ and, hence, $\mathcal{F}(A)=A$. Next, if $A=L_{\infty}(\Omega, \mu)$, with $\mu$ a $\sigma$-finite diffuse measure on a $\sigma$-algebra, then there exist no algebraically relatively strictly positive elements in $A$ and, hence, $\mathcal{F}(A)=\{0\}$.

If we will assume that the residue $a_{-1}$ is a minimal idempotent of $A$ instead of $a_{-1} \in \mathcal{F}(A)$ then Axiom $\left(\mathbf{A}_{\mathrm{LS}}\right)$ is valid for the algebra $B(E)$, where $E$ is an arbitrary Banach lattice. Unfortunately, the residue $a_{-1}$ of the resolvent $R(\cdot, a)$ of an irreducible element $a$ which belongs to an ordered Banach algebra $A$ satisfying Axiom $\left(\mathbf{A}_{1}\right)$ need not be a minimal idempotent (see [4]). Therefore, under this assumption, Axiom $\left(\mathbf{A}_{\mathrm{LS}}\right)$ is employed only for a narrow class of ordered Banach algebras. Moreover, as the next example shows, Axiom $\left(\mathbf{A}_{\mathrm{LS}}^{\prime}\right)$ in its present form and Axiom $\left(\mathbf{A}_{\mathrm{LS}}\right)$ with the assumption about the minimality of $a_{-1}$ do not hold in general for an arbitrary ordered Banach algebra $A$. This example also shows that the peripheral spectrum $\sigma_{\text {per }}(a)$ of an irreducible element a need not be cyclic while $r(a)$ is a pole of $R(\cdot, a)$ and the residue $a_{-1} \gg 0$.

Example 19. Consider the space $\ell_{\infty}$ of all bounded sequences $x=\left(x_{1}, x_{2}, \ldots\right)$. Under the natural algebraic operations, multiplication, and sup-norm, this space is a commutative Banach algebra with unit $\mathbf{e}=(1,1, \ldots)$. Fix an arbitrary number $\lambda_{0} \in \mathbb{C}$ and a sequence $\left\{z_{n}\right\}$ in $\mathbb{C}$ satisfying $\left|z_{n}\right|=1, z_{n} \neq \lambda_{0}$ for all $n, \lambda_{0} \neq 1, z_{1}=1, z_{n} \neq z_{m}$ for all $n \neq m$, and $z_{n} \rightarrow \lambda_{0}$ as $n \rightarrow \infty$. Define the sequence $z \in \ell_{\infty}$ by $z=\left(z_{1}, z_{2}, \ldots\right)$ and consider the algebraic wedge $K_{0}$ generated by e and $z$, i.e.,

$$
K_{0}=\left\{\sum_{j=0}^{n} \alpha_{j} z^{j}: n \in \mathbb{N} \text { and } \alpha_{j} \in \mathbb{R}^{+} \text {for all } j=0,1, \ldots, n\right\} .
$$

We claim that the closure $\overline{K_{0}}$ of $K_{0}$ is a normal cone. Indeed, for arbitrary $y=\sum_{j=0}^{n} \alpha_{j} z^{j}$ with $\alpha_{j} \in \mathbb{R}$ the inequalities $\left|\sum_{j=0}^{n} \alpha_{j}\right| \leq\|y\|_{\ell_{\infty}} \leq \sum_{j=0}^{n}\left|\alpha_{j}\right|$ holds as $z_{1}=1$. Therefore, if $\alpha_{j} \geq 0$ for $j=0,1, \ldots, n$ then

$$
\|y\|_{\ell_{\infty}}=\sum_{j=0}^{n} \alpha_{j}
$$

Let $x \in \ell_{\infty}$ and let $\pm x \in \overline{K_{0}}$. There exist two sequences $\left\{x_{n}\right\}$ and $\left\{y_{n}\right\}$ in $K_{0}$ satisfying $x_{n} \rightarrow x$ and $y_{n} \rightarrow-x$ and, hence, $x_{n}+y_{n} \rightarrow 0$ as $n \rightarrow \infty$. Let $x_{n}=\sum_{j=0}^{k_{n}} \alpha_{n j} z^{j}$ and $y_{n}=\sum_{j=0}^{k_{n}} \beta_{n j} z^{j}$, where $\alpha_{n j}, \beta_{n j} \geq 0$ for all $n$ and $j=1, \ldots, k_{n}$. Then $\sum_{j=0}^{k_{n}}\left(\alpha_{n j}+\beta_{n j}\right) z^{j} \rightarrow 0$ and so $0 \leq \sum_{j=0}^{k_{n}} \alpha_{n j} \leq \sum_{j=0}^{k_{n}}\left(\alpha_{n j}+\beta_{n j}\right) \rightarrow 0$ as $n \rightarrow \infty$. Taking into account the identity (21), we have $\left\|x_{n}\right\|_{\ell_{\infty}}=\sum_{j=0}^{n} \alpha_{n j} \rightarrow 0$. Therefore, $x=0$. Thus, $K=\overline{K_{0}}$ is a cone and, under the order induced by $K, \ell_{\infty}$ is an ordered Banach algebra. In view of the relation $z_{n} \neq z_{m}$ with $n \neq m$, the system $\left\{\mathbf{e}, z, z^{2}, \ldots\right\}$ is linearly independent and, in particular, every element $w \in K_{0}$ has a unique representation in the form $w=\sum_{j=0}^{n} \omega_{j} z^{j}$ with $\omega_{j} \geq 0$. Now if $u=\sum_{j=0}^{m} \mu_{j} z^{j} \in K_{0}$ and 
$0 \leq_{K_{0}} w \leq_{K_{0}} u$ then $\|w\|_{\ell_{\infty}}=\sum_{j=0}^{n} \omega_{j} \leq \sum_{j=0}^{n} \mu_{j}=\|u\|_{\ell_{\infty}}$. Thus, $K_{0}$ is a normal cone and, hence [7, p. 81, Exercise 9], $K$ is normal. We mention at once that the relation $\overline{K-K} \neq \ell_{\infty}$ holds as the Banach space $\ell_{\infty}$ is not separable; in particular, $K$ is not generating. Obviously, $r(z)=1$ and $R(\lambda, z)=\left(\frac{1}{\lambda-z_{1}}, \frac{1}{\lambda-z_{2}}, \ldots\right)$ for all $\lambda \notin \sigma(z)=\overline{\left\{z_{1}, z_{2}, \ldots\right\}}$. In particular, $\xi=1$ is an isolated point of $\sigma(z)$. For $\lambda$ close to this point and $\lambda \neq 1$ the inequality $|\lambda-1| \leq\left|\lambda-z_{n}\right|$ holds for all $n$. Whence $\left|\frac{(\lambda-1)^{2}}{\lambda-z_{n}}\right| \leq|\lambda-1|$ and so $(\lambda-1)^{2}\|R(\lambda, z)\|_{\ell_{\infty}} \leq|\lambda-1| \rightarrow 0$ as $\lambda \rightarrow 1$. Thus, $\xi=1$ is a simple pole of $R(\cdot, z)$. Let $r=\inf _{n>1}\left|1-z_{n}\right|>0$. For arbitrary $\epsilon$ and $\lambda$ satisfying $0<\epsilon<r$ and $|\lambda-1|<\epsilon$, we have $\left|\lambda-z_{n}\right| \geq\left|1-z_{n}\right|-|\lambda-1| \geq r-\epsilon$ and, hence, $\left|\frac{\lambda-1}{\lambda-z_{n}}\right| \leq \frac{\epsilon}{r-\epsilon} \rightarrow 0$ as $\epsilon \rightarrow 0$. Consequently, $\lim _{\lambda \rightarrow 1}\left|\frac{\lambda-1}{\lambda-z_{n}}\right|=0$ uniformly in $n=2,3, \ldots$ and so the residue $R(\cdot, z)$ at the point $\xi=1$ is equal to $\mathbf{e}_{\mathbf{1}}=(1,0,0, \ldots)$. If we can verify the identity $\mathbf{O I}\left(\ell_{\infty}\right)=\{0, \mathbf{e}\}$ (under the order induced by $K$ ) then this means the validity of the relation $\mathbf{e}_{\mathbf{1}} \gg 0$ while the point $\lambda_{0}$ is not an isolated point of the spectrum $\sigma(z)$. To this end, let the sequence $p \in \mathbf{O I}\left(\ell_{\infty}\right)$. Then $p$ is a characteristic function $\chi_{A}$ of a subset $A$ of $\mathbb{N}$ which has a representation in the form $p=\sum_{j=0}^{n} \theta_{j} z^{j}$ with $\theta_{j} \geq 0$. If $1 \notin A$ then $\left(\chi_{A}\right)_{1}=0$, whence $\sum_{j=0}^{n} \theta_{j}=0$ or $p=0$. If $1 \in A$ then $1 \notin \mathbb{N} \backslash A$, whence $p=\mathbf{e}$, as required. Obviously, the residue $\mathbf{e}_{1}$ is a minimal idempotent of the algebra $\ell_{\infty}$. Moreover, as is easy to see, we can choose the sequence $\left\{z_{n}\right\}$ such that the peripheral spectrum $\sigma_{\text {per }}(z)$ of the irreducible element $z$ is not cyclic.

Now, assuming the Lotz-Schaefer axiom $\left(\mathbf{A}_{\mathrm{LS}}\right)$, we continue the study of the peripheral spectrum and, in particular, will obtain some consequences of Theorem 12 .

Before, we discuss the following property of the algebra $B(E)$, where $E$ is a complex Banach lattice being the complexification of the real Banach lattice $E_{\mathbb{R}}$. Let $\left\{S_{n}\right\}$ and $\left\{T_{n}\right\}$ be two sequences in the space $B_{\mathrm{r}}(E)=B_{\mathrm{r}}\left(E_{\mathbb{R}}\right)$ of all regular operators on $E$ such that the sequence $\left\{S_{n}+i T_{n}\right\}$ converges in $B(E)$. Then $\left\{S_{n}\right\}$ and $\left\{T_{n}\right\}$ are also convergent. Indeed, fix a number $\epsilon>0$ and find an index $k \in \mathbb{N}$ satisfying $\left\|S_{n}-S_{m}+i\left(T_{n}-T_{m}\right)\right\|_{B(E)}<\epsilon$ for all $n, m \geq k$. Using the condition $S_{n} \in B_{\mathrm{r}}(E)$ and the inequality $\|y+i z\|_{E} \geq \max \left\{\|y\|_{E},\|z\|_{E}\right\}$ for all $y, z \in E_{\mathbb{R}}$, we have

$$
\left\|\left(S_{n}-S_{m}\right) x\right\|_{E} \leq\left\|\left(S_{n}-S_{m}\right) x+i\left(T_{n}-T_{m}\right) x\right\|_{E}<\epsilon
$$

for an arbitrary element $x \in E_{\mathbb{R}}$ with $\|x\|_{E}=\|x\|_{E_{\mathbb{R}}}=1$. Whence $\left\|S_{n}-S_{m}\right\|_{B\left(E_{\mathbb{R}}\right)}<\epsilon$ and so $\left\|S_{n}-S_{m}\right\|_{B(E)}<2 \epsilon$. Thus, the sequence $\left\{S_{n}\right\}$ is convergent; the case of $\left\{T_{n}\right\}$ is analogous. We axiomatize this property and make the next assumption:

(A) The convergence of the sequence $\left\{b_{n}+i c_{n}\right\}$, where $\left\{b_{n}\right\}$ and $\left\{c_{n}\right\}$ are two sequences in $A_{\mathrm{r}}$, implies the convergence of $\left\{b_{n}\right\}$ and $\left\{c_{n}\right\}$.

Evidently, if an ordered Banach algebra $A$ satisfies Axiom $\left(\mathbf{A}_{5}\right)$ then the algebra $A(q)$ also satisfies this axiom for every non-zero order idempotent $q$ of $A$. Next, as the example of the ordered Banach algebra $C^{1}[a, b]$ of all complex functions $x$ represented in the form $x=x_{1}+i x_{2}$, where the functions $x_{1}, x_{2}:[a, b] \rightarrow \mathbb{R}$ are continuously differentiable, under the natural algebraic operations, multiplication, order, and norm $\|x\|_{C^{1}[a, b]}=\max _{t \in[a, b]}|x(t)|+\max _{t \in[a, b]}|\dot{x}(t)|$, shows, Axiom $\left(\mathbf{A}_{5}\right)$ does not imply the normality of a cone $A^{+}$. 
Theorem 20. Let an ordered Banach algebra A satisfy Axioms $\left(\mathbf{A}_{1}\right)-\left(\mathbf{A}_{5}\right)$ and $\left(\mathbf{A}_{\mathrm{LS}}^{\prime}\right)$. Let an element a of $A$ be non-zero, order continuous, and irreducible. Let the point $r(a)$ be a pole of $R(\cdot, a)$. Then the peripheral spectrum $\sigma_{\mathrm{per}}(a)$ consists entirely of poles of $R(\cdot, a)$ and has the form $\sigma_{\mathrm{per}}(a)=r(a) H_{m}$ for some $m \in \mathbb{N}$.

Proof. In view of Theorem 12, it suffices to establish the inclusion $\sigma_{\text {per }}(a) \subseteq \sigma_{\mathrm{j}}\left(a ; a_{-1}\right)$. To this end, let $\lambda_{0} \in \sigma_{\text {per }}(a)$. Since $A$ satisfies Axiom $\left(\mathbf{A}_{\mathrm{LS}}^{\prime}\right), \lambda_{0}$ is a pole of $R(\cdot, a)$ of order $k$. The relations $a a_{\lambda_{0},-k}=a_{\lambda_{0},-k} a=\lambda_{0} a$ hold. We claim that $k=1$ and the residue $a_{\lambda_{0},-1}$ has the representation in the form $a_{\lambda_{0},-1}=z^{\prime}+i z^{\prime \prime}$, where the elements $z^{\prime}, z^{\prime \prime} \in A_{a_{-1}}$. The last two assertions imply the inclusion $\lambda_{0} \in \sigma_{\mathrm{j}}\left(a ; a_{-1}\right)$. To check them, let $\lambda_{0}=r(a)(\cos \varphi+i \sin \varphi)$ with $\varphi \in[0,2 \pi)$. We have the equalities

$$
\begin{gathered}
a_{\lambda_{0},-k}=\lim _{\lambda \rightarrow \lambda_{0}}\left(\lambda-\lambda_{0}\right)^{k} R(\lambda, a)=\lim _{t \downarrow 1}\left(t \lambda_{0}-\lambda_{0}\right)^{k} R\left(t \lambda_{0}, a\right)=\lim _{t \downarrow 1}(t-1)^{k} \lambda_{0}^{k} \sum_{j=0}^{\infty} \frac{1}{\left(t \lambda_{0}\right)^{j+1}} a^{j}= \\
=\lim _{t \downarrow 1}(t-1)^{k} \sum_{j=0}^{\infty} \frac{\cos ((k-j+1) \varphi)+i \sin ((k-j+1) \varphi)}{t^{j+1}} a^{j} .
\end{gathered}
$$

Fix an arbitrary sequence $\left\{t_{n}\right\}$ in $\mathbb{R}$ satisfying $t_{n} \downarrow 1$ and put

$$
b_{n}=\left(t_{n}-1\right)^{k} \sum_{j=0}^{\infty} \frac{\cos ((k-j+1) \varphi)}{t_{n}^{j+1}} a^{j} \text { and } c_{n}=\left(t_{n}-1\right)^{k} \sum_{j=0}^{\infty} \frac{\sin ((k-j+1) \varphi)}{t_{n}^{j+1}} a^{j} .
$$

Obviously, $b_{n}+i c_{n} \rightarrow a_{\lambda_{0},-k}$ as $n \rightarrow \infty$ and $\pm b_{n} \leq\left(t_{n}-1\right)^{k} \sum_{j=0}^{\infty} \frac{1}{t_{n}^{j+1}} a^{j} \rightarrow a_{-k}$; analogously, for $\left\{c_{n}\right\}$. Taking into account Axiom $\left(\mathbf{A}_{5}\right)$, we conclude the convergence of $\left\{b_{n}\right\}$ and $\left\{c_{n}\right\}$ and the validity of the representation $a_{\lambda_{0},-k}=x^{\prime}+i x^{\prime \prime}$, where $-a_{-k} \leq x^{\prime}, x^{\prime \prime} \leq a_{-k}$. If $k>1$ then $a_{-k}=0$ and, hence, $x^{\prime}, x^{\prime \prime}=0$ or $a_{\lambda_{0},-k}=0$, a contradiction. Thus, $k=1$, as required.

Lemma 21. If $A$ satisfies Axiom $\left(\mathbf{A}_{\mathrm{LS}}\right)$ then $A(q)$ also satisfies this axiom for every non-zero $q \in \mathbf{O I}(A)$.

Proof. Let $0 \leq a \in A(q)$, let $r(a)$ be a pole of $R_{q}(\cdot, a)$, where $R_{q}(\cdot, a)$ is a resolvent of $a$ in $A(q)$, and let the residue $a_{-1}$ of $R_{q}(\cdot, a)$ at the point $r(a)$ satisfy the condition $a_{-1} \in \mathcal{F}(A(q))$. We can assume $r(a)>0$. The inclusions [4]

$$
\rho_{\infty}(a ; A) \subseteq \rho(a ; A(q)) \text { and } \rho(a ; A(q)) \backslash\{0\} \subseteq \rho(a ; A)
$$

hold, where $\rho_{\infty}(a ; A)$ is the unbounded connected component of $\rho(a ; A)$. Moreover, for arbitrary $\lambda \in \rho(a ; A(q)) \backslash\{0\}$, we have the identity [4]

$$
R(\lambda, a)=R_{q}(\lambda, a)+\frac{1}{\lambda} q^{\mathrm{d}} .
$$

Thus, $r(a)$ is a pole of $R(\cdot, a)$ and the residues of $R(\cdot, a)$ and $R_{q}(\cdot, a)$ at $r(a)$ coincide. In view of the inclusion $\mathcal{F}(A(q)) \subseteq \mathcal{F}(A), a_{-1} \in \mathcal{F}(A)$. If $\lambda \in \sigma_{\text {per }}(a ; A(q))$ then $\lambda \in \sigma_{\text {per }}(a ; A)$ and, hence, $\lambda$ is a pole of $R(\cdot, a)$. Taking into account the first inclusion of (22), we infer that $\lambda$ is an isolated point of $\sigma(a ; A(q))$. Consequently, in view of (23), $\lambda$ is a pole of $R_{q}(\cdot, a)$.

The next result which follows easily from Corollary 5, Theorem 20, the inclusions (22), and the preceding lemma, characterizes the peripheral spectrum of a wide class of positive elements. 
Corollary 22. Let A satisfy Axioms $\left(\mathbf{A}_{1}\right),\left(\mathbf{A}_{3}\right)-\left(\mathbf{A}_{5}\right)$, and $\left(\mathbf{A}_{\mathrm{LS}}\right)$, let the algebra $A(q)$ satisfy Axiom $\left(\mathbf{A}_{2}\right)$ for every non-zero $q \in \mathrm{OI}(A)$, and let an element $a \in A_{\mathrm{n}}$ such that it has the Frobenius normal form and $r(a)$ is a pole of $R(\cdot, a)$. Then the identity $\sigma_{\mathrm{per}}(a)=r(a) \bigcup_{s=1}^{n} H_{m_{s}}$ holds with some $m_{1}, \ldots, m_{n} \in \mathbb{N}$.

As the next theorem shows, Axiom $\left(\mathbf{A}_{\mathrm{LS}}\right)$ can be employed to positive elements having the Frobenius normal form. Moreover, this result, Theorem 20, and the preceding corollary illustrate the importance of the notion of finite-rank element as we define it above. It also shows that our definition in the abstract case is the right one to use.

Theorem 23. Let an ordered Banach algebra A satisfy Axiom $\left(\mathbf{A}_{1}\right)$. Let an element $a \in A_{\mathrm{n}}$ have the Frobenius normal form. If $r(a)$ is a pole of $R(\cdot, a)$ then the residue $a_{-1}$ is a finite-rank element.

Proof. The idea is borrowed from the proof of the implication $(\mathbf{d}) \Longrightarrow(\mathbf{c})$ of Theorem 2.14 in [4]. Let order idempotents $p_{0}, p_{1}, \ldots, p_{n}, \mathbf{e}=p_{n} \geq \ldots \geq p_{0}=0$, determine the Frobenius normal form of $a$. Put $q_{j}=p_{j} p_{j-1}^{\mathrm{d}}$ for $j=1, \ldots, n$. If $r(a)=0$ then $r\left(a_{q_{j}}\right)=0$ and $a_{q_{j}}$ is irreducible with respect $q_{j}$ for all $j$, whence $a_{q_{j}}=0$. Thus, $\mathbf{O I}(A) \cap\left[0, q_{j}\right]=\left\{0, q_{j}\right\}$ and so $q_{j} \gg_{q_{j}}$. On the other hand, $a_{-1}=\mathbf{e}=\sum_{j=1}^{n} q_{j} \in \mathcal{F}(A)$.

Now we can suppose $r(a)>0$. For the proof, we use induction on $n$. For $n=1$ the element $a$ is irreducible and it remains to use Theorem $6(\mathbf{c})$. Next, assume that the desired assertion is proved if a parameter of the induction lies between 1 and $n-1 \geq 1$. Let us verify our assertion for $n$.

We consider first the case of the identity $r\left(a_{p_{n-1}}\right)=r(a)$ and show the inclusion

$$
\left(a_{-1}\right)_{p_{n-1}} \in \mathcal{F}(A) \text {. }
$$

The relation [4] $\left(a_{p_{n-1}}\right)_{-1}=\left(a_{-1}\right)_{p_{n-1}}$ holds in $A$. If $r(a) \notin \sigma\left(a_{p_{n-1}}\right)$ then $\left(a_{p_{n-1}}\right)_{-1}=0$ and (24) is obvious. Let $r(a) \notin \sigma\left(a_{p_{n-1}}\right)$. Then [4] $r(a)$ is a pole of $R_{p_{n-1}}\left(\cdot, a_{p_{n-1}}\right)$ in $A\left(p_{n-1}\right)$ (see the proof of Lemma 21). The order idempotents $p_{n-1}, \ldots, p_{0}$ are $a_{p_{n-1}}$ invariant. If $r\left(a_{q_{j}}\right)=r\left(a_{p_{n-1}}\right)$ then $a_{q_{j}}$ is irreducible with respect $q_{j}$ in $A$ and so in $A\left(p_{n-1}\right)$. By the induction hypothesis, the residue $\left(a_{p_{n-1}}\right)_{-1} \in \mathcal{F}\left(A\left(p_{n-1}\right)\right)$ (see the proof of Lemma 21 once more) and, hence, $\left(a_{p_{n-1}}\right)_{-1} \in \mathcal{F}(A)$.

Consider the case of the identity $r\left(a_{p_{n-1}^{\mathrm{d}}}\right)=r(a)$ and show the inclusion

$$
\left(a_{-1}\right)_{p_{n-1}^{\mathrm{d}}} \in \mathcal{F}(A) .
$$

Assuming without loss of generality that $r(a) \in \sigma\left(a_{p_{n-1}}^{\mathrm{d}}\right)$, we have the relations

$$
\left(a_{-1}\right)_{p_{n-1}^{\mathrm{d}}}=\left(a_{p_{n-1}^{\mathrm{d}}}\right)_{-1} \in \mathcal{F}\left(A\left(p_{n-1}^{\mathrm{d}}\right)\right) \subseteq \mathcal{F}(A) .
$$

Both inequalities $r\left(a_{p_{n-1}}\right) \leq r(a)$ and $r\left(a_{p_{n-1}^{\mathrm{d}}}\right) \leq r(a)$ cannot be strict simultaneously. To complete the proof, we consider three possible cases.

Case 1: $r\left(a_{p_{n-1}}\right)=r\left(a_{p_{n-1}^{\mathrm{d}}}\right)=r(a)$. As was shown above, the inclusions (24) and (25) hold. For $\lambda \in \rho(a ; A) \backslash\{0\}$, we have the identity [4]

$$
p_{n-1} R(\lambda, a) p_{n-1}^{\mathrm{d}}=R\left(\lambda, a_{p_{n-1}}\right) p_{n-1} a p_{n-1}^{\mathrm{d}} R\left(\lambda, a_{p_{n-1}^{\mathrm{d}}}\right)
$$


which implies

$$
p_{n-1} a p_{n-1}^{\mathrm{d}}=\left(a_{p_{n-1}}\right)_{-1} p_{n-1} a p_{n-1}^{\mathrm{d}}\left(a_{p_{n-1}^{\mathrm{d}}}\right)_{0}+\left(a_{p_{n-1}}\right)_{0} p_{n-1} a p_{n-1}^{\mathrm{d}}\left(a_{p_{n-1}^{\mathrm{d}}}\right)_{-1} \in \mathcal{F}(A)
$$

as $\mathcal{F}(A)$ is an algebraic ideal. Using the $a_{-1}$-invariance of $p_{n-1}$, we obtain $a_{-1} \in \mathcal{F}(A)$.

Case 2: $r\left(a_{p_{n-1}}\right)=r(a)$ and $r\left(a_{p_{n-1}^{\mathrm{d}}}\right)<r(a)$. Then (24) holds. Moreover, we have [4] $p_{n-1}^{\mathrm{d}} a_{-1}=0$. Hence, taking into account (26), we conclude $a_{-1} \in \mathcal{F}(A)$.

Case 3: $r\left(a_{p_{n-1}}\right)<r(a)$ and $r\left(a_{p_{n-1}^{\mathrm{d}}}\right)=r(a)$. Then (25) holds. Moreover, we have [4] $a_{-1} p_{n-1}=0$. Hence, taking into account (26), we conclude $a_{-1} \in \mathcal{F}(A)$.

We mention the following important consequence of the preceding theorem which shows once more that the definition of an $f$-pole is the right and natural one to use (see [4], where the detail discussion of this notion can be found).

Corollary 24. Let $A$ be a Dedekind complete and let $a \in A$ be a spectrally order continuous element with $r(a)>0$. If $r(a)$ is a finite-rank pole of $R(\cdot, a)$ then the residue $a_{-1}$ is a finite-rank element.

It is not known if the point $r(a)$ is an $f$-pole of $R(\cdot, a)$ of an arbitrary irreducible element a of $A$ such that $r(a)$ is a pole of $R(\cdot, a)$ (of course, under the assumptions of Theorem 6 ). As can be shown (see [4]), the affirmative answer to this question is equivalent to: $0 \leq b<a$ implies $r(b)<r(a)$, which will be discussed in Section 5. In particular, it is not known if the converse to the preceding corollary is true.

We now turn our attention to the conditions of the primitivity of irreducible element $a$ in an ordered Banach algebra $A$. Recall that an element $b$ of an arbitrary Banach algebra $B$ is called primitive if the peripheral spectrum $\sigma_{\text {per }}(b)$ contains at most one point; all other elements of $B$ are called imprimitive.

We begin with the next criteria of the primitivity.

Theorem 25. Let an ordered Banach algebra A satisfy Axioms $\left(\mathbf{A}_{1}\right)-\left(\mathbf{A}_{5}\right)$ and $\left(\mathbf{A}_{\mathrm{LS}}\right)$. Let a $\in A$ be a non-zero irreducible element such that $r(a)$ is a pole of $R(\cdot, a)$. The following statements are equivalent:

(a) The element a is primitive;

(b) The element $a^{m}$ is primitive for all $m \in \mathbb{N}$;

(c) The element $a^{m}$ is irreducible for all $m \in \mathbb{N}$;

(d) The sequence $\left\{\left(\frac{a}{r(a)}\right)^{n}\right\}$ converges to an algebraically strictly positive element.

Proof. The implication (a) $\Longrightarrow$ (b) follows at once from the identity $\sigma\left(a^{m}\right)=f(\sigma(a))$ with $f(z)=z^{m}$ and the implication (b) $\Longrightarrow$ (a) is obvious.

(a) $\Longrightarrow$ (c) If $a^{m}$ is reducible for some $m \in \mathbb{N}$ then $m>1$ and, in view of Theorem 12, the points $r(a) e^{i \frac{2 \pi}{m^{\prime \prime}} j} \in \sigma(a)$ for all $j=0,1, \ldots, m^{\prime \prime}-1$ with $m^{\prime \prime}>1$, a contradiction.

(c) $\Longrightarrow$ (a) Proceeding by contradiction and using Axiom $\left(\mathbf{A}_{\mathrm{LS}}\right)$ and Theorem 20 , we conclude the validity of the identity $\sigma_{\text {per }}(a)=r(a) H_{k}$ with $k>1$. Taking into account Theorem 12 once more, we obtain the reducibility $a^{m}$ of for some $m>1$, which is impossible. 
(a) $\Longrightarrow$ (d) We shall prove first the next assertion: If $b$ is a primitive positive element of an arbitrary ordered Banach algebra $A_{0}$ such that $r(b)>0$ is a simple pole of $R(\cdot, b)$ then $\left(\frac{b}{r(b)}\right)^{n}$ converges to the residue $b_{-1}$ of $R(\cdot, b)$ at $r(b)$. Indeed, we define the element $c$ by $c=b-r(b) b_{-1}$. The Spectral Mapping Theorem yields $\sigma(c)=(\sigma(c) \cup\{0\}) \backslash\{r(b)\}$. Taking into account the primitivity of $b$, we infer $r(c)<r(b)$ or $r\left(\frac{c}{r(b)}\right)<1$. By the Gelfand formula, the equality $r\left(\frac{c}{r(b)}\right)=\lim _{n \rightarrow \infty} \frac{\left\|c^{n}\right\|_{A_{0}}}{r(b)^{n}}$ holds and, in particular, $\left(\frac{c}{r(b)}\right)^{n} \rightarrow 0$ as $n \rightarrow \infty$. Using the identities $b b_{-1}=b_{-1} b=r(b) b_{-1}$, we obtain the relations $b_{-1} c=c b_{-1}=0$. Therefore, $b^{n}=c^{n}+r(b)^{n} b_{-1}$ for all $n$. Consequently, the relations $\left(\frac{b}{r(b)}\right)^{n}=\left(\frac{c}{r(b)}\right)^{n}+b_{-1} \rightarrow b_{-1}$ hold, as required. Now it only remains to remember Theorem $6(\mathbf{b}),(\mathbf{c})$. According to it, $r(a)$ is a simple pole of $R(\cdot, A)$ and $a_{-1} \gg 0$ as $a$ is irreducible.

(d) $\Longrightarrow$ (c) If $a^{m}$ is reducible for some number $m>1$ then $p^{\mathrm{d}} a^{m} p=0$ for some non-trivial $p \in \mathbf{O I}(A)$. Evidently, $p^{\mathrm{d}} a^{m n} p=0$ for all $n \in \mathbb{N}$. Thus, $0=p^{\mathrm{d}}\left(\frac{a}{r(a)}\right)^{m n} p \rightarrow p^{\mathrm{d}} a_{-1} p$ as $n \rightarrow \infty$ and, hence, $p^{\mathrm{d}} a_{-1} p=0$. The latter contradicts to the algebraic strict positivity of $a_{-1}$.

A non-zero order idempotent $p$ of $A$ is called order minimal if the equality $\sum_{j=1}^{n} p_{j}=\mathbf{e}$, where $p_{j} \in \mathbf{O I}(A)$ for $j=1, \ldots, n$ and $p_{j^{\prime}} p_{j^{\prime \prime}}=0$ for $j^{\prime} \neq j^{\prime \prime}$, implies the existence of an index $j_{0}$ satisfying $p \leq p_{j_{0}}$.

Corollary 26. Under the assumptions of Theorem 25 each of the following conditions guarantees the primitivity of the element $a$ :

(a) The element $a \gg 0$;

(b) The element $a_{p}>0$ for some order minimal $p \in \mathbf{O I}(A)$.

Proof. (a) We shall show the irreducibility of $a^{m}$ for all $m \in \mathbb{N}$. In view of part (a) of the preceding theorem, the latter implies the desired assertion. To this end, we assume $q^{\mathrm{d}} a^{m} q=0$ for some $m>1$ and $q \in \mathbf{O I}(A)$. Since $A$ has a disjunctive product, there exists an order idempotent $q_{1}$ satisfying $q^{\mathrm{d} a} a q_{1}=q_{1}^{\mathrm{d}} a^{m-1} q=0$. We can suppose $q \neq \mathbf{e}$. Then $q_{1}=0$ and so $a^{m-1} q=0$. If $m>2$ then there exists an order idempotent $q_{2}$ satisfying $a q_{2}=q_{2}^{\mathrm{d}} a^{m-2} q=0$ and so $a^{m-2} q=0$. Finally, using a reverse finite induction, we obtain $a q=0$ or $q=0$, as required.

(b) Proceeding by contradiction and taking into account Theorems 25 and 12, we find $p_{1}, \ldots, p_{m} \in \mathbf{O I}(A)$ with $m>1$ determining the cyclic form of the element $a$ and, in particular, $p_{j} a=a p_{j+1}$ for all $j=1, \ldots, m$. In view of the order minimality of $p$, we choose an index $j_{0}$ satisfying $p \leq p_{j_{0}}$. Then the equalities $p a=p p_{j_{0}} a=p a p_{j_{0}+1}$ hold and, hence, $a_{p}=\operatorname{pap}_{j_{0}+1} p=0$, a contradiction.

If $b$ is an element of a Banach algebra $B$ with a unit $\mathbf{u}$ such that $r(b) \in \sigma(b)$ then $b+\lambda \mathbf{u}$ is primitive for all numbers $\lambda>0$. The next result makes more precisely this fact.

Corollary 27. Let A satisfy Axioms $\left(\mathbf{A}_{1}\right)-\left(\mathbf{A}_{5}\right)$ and let $a, b \in A^{+}$. If $a+b$ is irreducible, $b_{p}>0$ for all $0<p \in \mathbf{O I}(A)$, and $r(a+b)$ is a pole of $R(\cdot, a+b)$ then $a+b$ is primitive.

Proof. Again, proceeding by contradiction and taking into account Theorems 25] and 12 , we find $p_{1}, \ldots, p_{m} \in \mathbf{O I}(A)$ with $m>1$ determining the cyclic form of $a+b$ and, in particular, $p_{j}(a+b)=(a+b) p_{j+1}$ for all $j=1, \ldots, m$. Therefore, $0=p_{j}(a+b) p_{j}=a_{p_{j}}+b_{p_{j}}>0$, a contradiction. 
An element $a$ of $A$ is said to be symmetric whenever $p a q=q a p$ for all $p, q \in \mathbf{O I}(A)$.

Corollary 28. Suppose that all assumptions of Theorem 25 are satisfied and, in additional, the element $a$ is symmetric. Then the element $a$ is primitive if and only if $a+r(a) \mathbf{e}$ is invertible.

Proof. The necessity is obvious. We shall prove the sufficiency. Proceeding by contradiction, we find $p_{1}, \ldots, p_{m} \in \mathbf{O I}(A)$ with $m>1$ determining the cyclic form of $a$. In view of the validity of the implication $(\mathbf{b}) \Longrightarrow(\mathbf{c})$ of Theorem 12 and our condition, the natural number $m$ is odd. Then $p_{1} a p_{2}=a p_{2}>0$. Since $a$ is symmetric, $p_{2} a p_{1}>0$. On the other hand, $p_{2} a p_{1}=a p_{3} p_{1}=0$, a contradiction.

As follows from Theorem 25, if an element $a$ of $A$ is irreducible and primitive then $a^{m}$ is irreducible for all $m \in \mathbb{N}$. Unfortunately, the relation $a^{m} \gg 0$ need not hold for any $m$ in the case of an integral operator even (see [2]). Nevertheless, we have the next result.

Corollary 29. Suppose that all assumptions of Theorem 25 are satisfied and, in additional, the element $a$ is primitive. If $a^{m_{0}} \gg 0$ for some $m_{0} \in \mathbb{N}$ then $a^{m} \gg 0$ for all natural $m \geq m_{0}$.

Proof. Proceeding by contradiction, we find $m>m_{0}$ and non-zero $p, q \in \mathbf{O I}(A)$ satisfying $p a^{m} q=0$. Obviously, $p a^{m_{0}} a^{m-m_{0}} q=0$. Since the algebra $A$ has a disjunctive product, we have $p a^{m_{0}} p_{1}=p_{1}^{\mathrm{d}} a^{m-m_{0}} q=0$ for some $p_{1} \in \mathbf{O I}(A)$. According to our condition, $p_{1}=0$ and so $a^{m-m_{0}} q=0$. In view of Theorem 25 , the element $a^{m-m_{0}}$ is irreducible and, hence, $q=0$, a contradiction.

For the case of an arbitrary (not necessarily irreducible) element $a \in A^{+}$, we have the next.

Proposition 30. Let $A$ be an ordered Banach algebra. Let $a \in A$ be a positive element such that $r(a)$ is a pole of $R(\cdot, A)$ and every point $\alpha \in \sigma_{\text {per }}(a)$ is an eigenvalue of either the operator $L_{a}$ or the operator $R_{a}$ on $A$. The following statements hold:

(a) The sequence $a^{n} \rightarrow 0$ as $n \rightarrow \infty$ if and only if $r(a)<1$;

(b) If $r(a)=1$ then the sequence $\left\{a^{n}\right\}$ is convergent if and only if $r(a)$ is a simple pole of $R(\cdot, a)$ and the element $a$ is primitive;

(c) If $r(a)>1$ then the sequence $\left\{a^{n}\right\}$ is not convergent.

Proof. (b) Let $r(a)=1$ and let $\left\{a^{n}\right\}$ be convergent. Then $\left\|a^{n}\right\|_{A} \leq c$ for all $n$ and some constant $c \in \mathbb{R}^{+}$. For all $\lambda \in \mathbb{R}, \lambda>1$, we have

$$
\begin{gathered}
(\lambda-1)^{2}\|R(\lambda, a)\|_{A}=(\lambda-1)^{2}\left\|\sum_{j=0}^{\infty} \frac{1}{\lambda^{j+1}} a^{j}\right\|_{A} \leq \\
(\lambda-1)^{2} \sum_{j=0}^{\infty} \frac{1}{\lambda^{j+1}}\left\|a^{j}\right\|_{A} \leq C(\lambda-1)^{2} \sum_{j=0}^{\infty} \frac{1}{\lambda^{j+1}}=C(\lambda-1)^{2} \frac{\lambda}{\lambda-1}=C \lambda(\lambda-1) \rightarrow 0
\end{gathered}
$$

as $\lambda \downarrow 1$. Thus, $r(a)$ is a simple pole of $R(\cdot, a)$. Now we consider $\alpha \in \sigma_{\text {per }}(a)$. In view of our condition, $\alpha$ is an eigenvalue of $L_{a}$ (the case of $R_{a}$ is analogous), i.e., $a b=\alpha b$ for some nonzero $b \in A$. Obviously, $a^{n} b=\alpha^{n} b$ for all $n \in \mathbb{N}$ and the sequence $\left\{\alpha^{n} b\right\}$ converges. Therefore, $\left\{\alpha^{n}\right\}$ converges. The latter is possible for the case of $\alpha=1$ only and, hence, $a$ is primitive. 
The converse assertion, namely, the relation $a^{n} \rightarrow a_{-1}$ as $n \rightarrow \infty$, was shown in the proof of the implication $(\mathbf{a}) \Longrightarrow$ (d) of Theorem 25 .

(a) The sufficiency is clear. We shall check the necessity. Assume that $a^{n} \rightarrow 0$ as $n \rightarrow \infty$. This implies $r(a) \leq 1$. If $r(a)=1$ then, as was mentioned above, $a^{n} \rightarrow a_{-1}$ and, hence, $a_{-1}=0$, a contradiction.

(c) If $r(a)=1$ then $\left\{a^{n}\right\}$ is unbounded and, in particular, is not convergent.

\section{Other viewpoints on the irreducibility and the primitivity}

Recall that an element $a \in A^{+}$is said to be irreducible whenever the equality $p^{\mathrm{d}} a p=0$, where $p \in \mathbf{O I}(A)$, implies $p=0$ or $p=\mathbf{e}$. Irreducible elements were introduced in [10] for the case of Banach lattice algebras and in [4] for the case of ordered Banach algebras. Under the natural assumptions, these elements have nice spectral properties and, under such a notion of irreducibility, the theorem about the Frobenius normal form holds. Nevertheless, the purpose of this section is to establish some results which allow us in a new fashion to take a glance at the algebraic nature of such notions as the irreducibility and the primitivity and, thus, to break some ground for further research of these notions in an ordered Banach algebras.

Let $E$ be a Dedekind complete Banach lattice. As usual, we denote the set of all operators on $E$ of the form $\sum_{j=1}^{n} f_{j} \otimes x_{j}$, where $f_{j} \in E_{\mathrm{n}}^{\sim}, x_{j} \in E$, and $\left(f_{j} \otimes x_{j}\right) x=\left(f_{j}(x)\right) x_{j}$ for all $j=1, \ldots, n$ and $x \in E$, by $E_{\mathrm{n}}^{\sim} \otimes E$. The band $\left(E_{\mathrm{n}}^{\sim} \otimes E\right)^{\text {dd }}$ generated by $E_{\mathrm{n}}^{\sim} \otimes E$ in the Banach lattice $L_{\mathrm{r}}(E)$ with the $r$-norm $\|T\|_{r}=\|T\|_{B(E)}$ is called [1, p. 193] the band of abstract integral operators. As is easy to see, $\left(E_{\mathrm{n}}^{\sim} \otimes E\right)^{\mathrm{dd}} \subseteq L_{\mathrm{n}}(E)$ and if $T \in L_{\mathrm{r}}(E)$ and $S \in\left(E_{\mathrm{n}}^{\sim} \otimes E\right)^{\mathrm{dd}}$ then $T S, S T \in\left(E_{\mathrm{n}}^{\sim} \otimes E\right)^{\mathrm{dd}}$. In particular, under the $r$-norm, $\left(E_{\mathrm{n}}^{\sim} \otimes E\right)^{\mathrm{dd}}$ is an ordered Banach algebra (possibly, without a unit). If $E$ is a function space and $E_{\mathrm{n}}^{\sim}$ separates points of $E$ then, by the Lozanovsky theorem [1, p. 199], $\left(E_{\mathrm{n}}^{\sim} \otimes E\right)^{\text {dd }}$ coincides with the band of regular integral operators on $E$. Next, as can be shown (see [17]), if an arbitrary Banach lattice $E$ possesses the non-trivial band $E_{\mathrm{c}}^{\sim}$ of all $\sigma$-order continuous functionals, i.e., $E_{\mathrm{c}}^{\sim} \neq\{\emptyset\}$, and admitts a $\sigma$-order continuous irreducible operator $T$ then $E_{\mathrm{n}}^{\sim}=E_{\mathrm{c}}^{\sim}$ and $E_{\mathrm{n}}^{\sim}$ separates the points of $E$. We also recall that in a Banach algebra $B$ with or without a unit the wedge operator $W_{b}$ on $B$ [8, pp. 17, 70], where $b \in B$, is defined by $W_{b}=b a b$ for $a \in B$. As is easy to see, $r\left(W_{b}\right) \leq r(b)^{2}$ (we put $r(b)=\lim _{n \rightarrow \infty}\left\|b^{n}\right\|_{B}^{\frac{1}{n}}$ if $B$ does not have a unit).

Theorem 31. Let $E$ be a Dedekind complete Banach lattice such that $E_{\mathrm{n}}^{\sim}$ separates points of E. Let $T$ be a non-zero positive order continuous operator on $E$ such that $r(T)$ is a pole of $R(\cdot, T)$. If the restriction of the wedge operator $W_{T}$ to $\left(E_{\mathrm{n}}^{\sim} \otimes E\right)^{\mathrm{dd}}$ is an irreducible operator then two operators $T$ and $T^{\prime}$ are also irreducible and, moreover, primitive, where $T^{\prime}$ is the restriction of the adjoint operator $T^{*}$ to $E_{\mathrm{n}}^{\sim}$.

Proof. Consider a $T$-invariant band $B \neq\{0\}$. Fix $\lambda>r(T)$. Using the $R(\lambda, T)$-invariance of this band and the inequality $T R(\lambda, T) \leq \lambda R(\lambda, T)$, we find a non-zero element $z_{0} \in B^{+}$ satisfying $T z_{0} \leq \lambda z_{0}$. For an arbitrary non-zero functional $h_{0} \in\left(E^{*}\right)^{+}$such that $T^{*} h_{0} \leq \lambda h_{0}$, we have $W_{T}\left(h_{0} \otimes z_{0}\right) \leq \lambda^{2} h_{0} \otimes z_{0}$. As is easy to see, the operator $W_{T}$ is order continuous 
on $\left(E_{\mathrm{n}}^{\sim} \otimes E\right)^{\mathrm{dd}}$. Therefore, $h_{0} \otimes z_{0}$ is a weak order unit in $\left(E_{\mathrm{n}}^{\sim} \otimes E\right)^{\mathrm{dd}}$. If $z \in E^{+}$and $z_{0} \perp z$ then $\left(h \otimes z_{0}\right) \wedge(h \otimes z)=h \otimes\left(z_{0} \wedge z\right)=0$, whence $h \otimes z=0$ or $z=0$. Thus, $z_{0}$ is a weak order unit. Consequently, $B=E$ and the irreducibility of $T$ has been proved. In particular, $T^{\prime}$ is (see [2], the proof of Theorem 1) also irreducible. Moreover, there exist a weak order unit $x_{0} \in E$ and a strictly positive functional $f \in E_{\mathrm{n}}^{\sim}$ satisfying $T x_{0}=r(T) x_{0}$ and $T^{*} f_{0}=r(T) f_{0}$.

Now let us verify the primitivity of the operator $T$. In view of the inclusion [1, p. 256] $\rho_{\infty}\left(T^{*} ; B\left(E^{*}\right)\right) \subseteq \rho\left(T^{\prime} ; B\left(E_{\mathrm{n}}^{\sim}\right)\right)$, the latter implies the primitivity of $T^{\prime}$. Proceeding by contradiction, we find ([2]; see also part (b) of Theorem 12) elements $y_{1}, \ldots, y_{m}$, where $m>1$, satisfying $\sum_{j=1}^{m} y_{j}=x_{0}, y_{j^{\prime}} \wedge y_{j^{\prime \prime}}=0$ for $j^{\prime} \neq j^{\prime \prime}$, and $T y_{j+1}=r(T) y_{j}$ for $j=1, \ldots, m$. Define the functionals $g_{j} \in E^{*}$ via the formula $g_{j}=P_{y_{j}}^{*} f_{0}$, where $P_{y_{j}}$ is the order projection from $E$ onto the projection band $B_{y_{j}}$ generated by $y_{j}$. Obviously, $\sum_{j=1}^{m} g_{j}=f_{0}, g_{j^{\prime}} \perp g_{j^{\prime \prime}}$ for $j^{\prime} \neq j^{\prime \prime}$, and $g_{j} \in E_{\mathrm{n}}^{\sim}$. Taking into account the identities $T^{*} g_{j}=T^{*} P_{y_{j}}^{*} f_{0}=P_{y_{j+1}}^{*} T^{*} f_{0}$, we have the relation $T^{*} g_{j^{\prime}} \perp T^{*} g_{j^{\prime \prime}}$ for $j^{\prime} \neq j^{\prime \prime}$. Moreover, $\left(T^{*} g_{j}\right) y_{k}=g_{j}\left(y_{k-1}\right)=0$ for $k \neq j+1$, whence $\left(T^{*} g_{j}\right)\left(x_{0}-y_{j+1}\right)=g_{k} y_{j+1}=0$ and so $\left(T^{*} g_{j} \wedge g_{k}\right) x_{0}=0$. Since $x_{0}$ is a weak order unit, the last equality implies $T^{*} g_{j} \perp g_{k}$ for $k \neq j+1$. Consequently,

$$
T^{*} g_{j}-r(T) g_{j+1} \perp r(T) \sum_{\substack{n=1 \\ n \neq j+1}}^{m} g_{n}-\sum_{\substack{n=1 \\ n \neq j}}^{m} T^{*} g_{n} .
$$

On the other hand, $\sum_{n=1}^{m} T^{*} g_{n}=r(T) \sum_{n=1}^{m} g_{n}$ or

$$
T^{*} g_{j}-r(T) g_{j+1}=r(T) \sum_{\substack{n=1 \\ n \neq j+1}}^{m} g_{n}-\sum_{\substack{n=1 \\ n \neq j}}^{m} T^{*} g_{n}
$$

whence $T^{*} g_{j}=r(T) g_{j+1}$ for all $j=1, \ldots, m$. Put $f_{j}=g_{m-j+1}$. Evidently,

$$
T^{*} f_{j+1}=T^{*} g_{m-j}=r(T) g_{m-j+1}=r(T) f_{j} .
$$

Thus, $W_{T} \sum_{n=1}^{m} f_{n} \otimes y_{n}=r(T)^{2} \sum_{n=1}^{m} f_{n} \otimes y_{n}$. Therefore, $\sum_{n=1}^{m} f_{n} \otimes y_{n}$ is a weak order unit in the band $\left(E_{\mathrm{n}}^{\sim} \otimes E\right)^{\mathrm{dd}}$. This contradicts to the relation $f_{j} \otimes y_{k} \perp \sum_{n=1}^{m} f_{n} \otimes y_{n}$ for all $j \neq k$.

It is not known if the converse to the assertion of the preceding theorem holds. Nevertheless, as the next result shows, we can assert the converse in the case of the Banach algebra $M_{n}(\mathbb{C})$ of all $n \times n$ matrices with complex entries and the natural multiplication and order. We mention first that a matrix $A \in M_{n}(\mathbb{C})$ is irreducible if and only if the transpose $A^{t}$ of $A$ is irreducible.

Theorem 32. Let $T$ be an $n \times n$ positive matrix. Then $T$ is irreducible and primitive if and only if the wedge operator $W_{T}$ on $M_{n}(\mathbb{C})$ is irreducible.

Proof. In view of Theorem 31 , it is enough to verify the necessity. Clearly, we can assume $n>1$. Let the inequality $W_{T} S \leq \lambda S$ hold for a number $\lambda \geq 0$ and a non-zero positive matrix $S \in M_{n}(\mathbb{C})$. Since the matrix $T$ is primitive, $T^{k}$ is strongly positive for some $k \in \mathbb{N}$ (see 
Theorem 25], i.e., all its entries are strictly positive. Therefore, $\lambda^{k} S \geq W_{T}^{k} S=T^{k} S T^{k}$ and so $S$ is also strongly positive. Finally, $W_{T}$ is irreducible.

The following result suggests another approach to the notion of irreducibility in ordered Banach algebras.

Theorem 33. Let $T$ be an $n \times n$ matrix. Then $T$ is irreducible if and only if the operator $L_{T}+R_{T}$ on $M_{n}(\mathbb{C})$ is irreducible.

Proof. Necessity. We recall first that (see the equalities (5)) $\left(L_{T}+R_{T}\right) Q=T Q+Q T$, where $Q \in M_{n}(\mathbb{C})$. Let the inequality $L_{T} S \leq \lambda S$ holds for a number $\lambda \geq 0$ and a non-zero positive matrix $S \in M_{n}(\mathbb{C}), S=\left[s_{i j}\right]$. There exist indexes $i_{0}, j_{0}=1, \ldots, n$ such that the entry $s_{i_{0} j_{0}}>0$. If $s^{j_{0}}$ is the $j_{0}^{\text {th }}$ column of $S$ then, in view of the inequality $T S \leq \lambda S$, we have $T s^{j_{0}} \leq \lambda s^{j_{0}}$. Taking into account the irreducibility of $T$, we obtain $s_{i j_{0}}>0$ for all $i=1, \ldots, n$. On the other hand, the inequality $S^{t} T^{t} \leq \lambda S^{t}$ holds, $T^{t}$ is irreducible, and $s_{j_{0} i}^{t_{0}}>0$ for all $i$, where $S^{t}=\left[s_{i j}^{t}\right]$. As was shown above, $s_{j i}^{t}>0$ or $s_{i j}>0$ for all $i, j$, and we are done.

Sufficiency. Let the operator $L_{T}+R_{T}$ be irreducible. In particular, $L_{T}+R_{T} \geq 0$. Then $0 \leq\left(L_{T}+R_{T}\right) I=2 T$ or $T \geq 0$. Assume that $T$ is reducible. Then for some $k=1, \ldots, n-1$ there exist indexes $j_{1}, \ldots, j_{k}$ satisfying $t_{i j}=0$ for all $i \notin J$ and $j \in J$, where $J=\left\{j_{1}, \ldots, j_{k}\right\}$. Consider the band $B$ in $M_{n}(\mathbb{C})$ defined by

$$
B=\left\{S \in M_{n}(\mathbb{C}): s_{i j}=0 \text { for all } i \notin J \text { and all } j\right\} .
$$

Let $S \in B$. If $T S=\left[(t s)_{i j}\right]$ then for $i \notin J$, we have

$$
(t s)_{i j}=\sum_{m=1}^{n} t_{i m} s_{m j}=\sum_{m \notin J} t_{i m} s_{m j}+\sum_{m \in J} t_{i m} s_{m j}=0 .
$$

If $S T=\left[(s t)_{i j}\right]$ then $(s t)_{i j}=0$ for $i \notin J$. Thus, the band $B$ is $L_{T}+R_{T}$-invariant, which is impossible.

\section{$5 \quad$ When does $0 \leq b<a$ imply $r(b)<r(a)$ ?}

Let $T$ be an order continuous irreducible operator on a Banach lattice $E$, let $r(T)$ be a pole of $R(\cdot, T)$ of order $k$, and let the coefficient $T_{-k}$ of the Laurent series expansion of $R(\cdot, T)$ around $r(T)$ also be order continuous (the latter holds if, e.g., the Lorenz seminorm $\|\cdot\|_{L}$ on $E$ is a norm). Then [4] the operator inequalities $0 \leq S<T$, where $S, T \in B(E)$, imply the spectral radius inequality $r(S)<r(T)$. In particular, if $S, T \in M_{n}(\mathbb{C}), T$ is irreducible, and $0 \leq S<T$ then $r(S)<r(T)$. As was mentioned above (see remarks after Corollary 24), the analogous question, i.e., the validity of the inequality $r(b)<r(a)$ where $0 \leq b<a$ and the element $a$ is irreducible, remains open in the case of an ordered Banach algebra. The purpose of the present section is to discuss a number of additional conditions under which the answer to this question is affirmative. We mention at once that in research of this problem the assumption that $r(a)$ is a pole of $R(\cdot, a)$ is natural absolutely and cannot be even reject in the case of operators (see [2, 3]).

Theorem 34. Let $A$ be a finite-dimensional ordered Banach algebra with a disjunctive product and let $a, b \in A$. If the element $a$ is irreducible then $0 \leq b<a$ implies $r(b)<r(a)$. 
Proof. We claim that all assumptions of Theorem 6 hold. Indeed, if elements $q_{j} \in \mathbf{O I}(A)$ with $j=1, \ldots, k$ and $q_{j^{\prime}} q_{j^{\prime \prime}}=0$ for $j^{\prime} \neq j^{\prime \prime}$ then $q_{1}, \ldots, q_{k}$ are linearly independent. Therefore, there exists a maximal collection of pairwise disjoint elements $\left\{p_{1}, \ldots, p_{n}\right\}$ in $\mathbf{O I}(A)$. Obviously, $\sum_{j=1}^{n} p_{j}=\mathbf{e}$. We have the identity

$$
\mathrm{OI}(A)=\left\{\sum_{j \in J} p_{j}: J \subseteq\{1, \ldots, n\}\right\} .
$$

Actually, if an order idempotent $p$ of $A$ satisfies $0<p p_{j}<p_{j}$ for some $j=1, \ldots, n$ then $0<p p_{j}<p_{j}$ and the collection $\left\{p_{1}, \ldots, p_{j-1}, p p_{j}, p^{\mathrm{d}} p_{j}, p_{j+1}, \ldots, p_{n}\right\}$ consists of pairwise disjoint elements. The latter contradicts to the maximality of $\left\{p_{1}, \ldots, p_{n}\right\}$ and, hence, either $p p_{j}=0$ or $p p_{j}=p_{j}$. Putting $J=\left\{j: p p_{j}>0\right\}$, we obtain $p=p \sum_{j=1}^{n} p_{j}=\sum_{j \in J} p p_{j}=\sum_{j \in J} p_{j}$, and (27) has been checked. In particular, the Boolean algebra $\mathrm{OI}(A)$ is Dedekind complete. Consider a net $\left\{b_{\alpha}\right\}$ satisfying $b_{\alpha} \downarrow 0$ in $\mathbf{O I}(A)$. As was shown above, $b_{\alpha}=\sum_{j \in J_{\alpha}} p_{j}$ for every $\alpha$, where $J_{\alpha} \subseteq\{1, \ldots, n\}$. Evidently, if $b_{\alpha^{\prime}}<b_{\alpha^{\prime \prime}}$ then $J_{\alpha^{\prime}} \subsetneq J_{\alpha^{\prime \prime}}$. From the latter, we conclude easily the existence of an index $\alpha_{0}$ such that $b_{\alpha_{0}}=0$. Thus, the equality $A^{+}=A_{\mathrm{n}}$ holds. On the other hand, as is well known, the spectrum $\sigma(x ; B)$ of every element $x$ of an arbitrary finitedimensional Banach algebra $B$ with a unit is finite and consists of poles of the resolvent $R(\cdot, x)$. Now it only remains to use part (d) of Theorem 6.

As the next example shows, the preceding theorem is not valid without the assumption about a disjunctive product.

Example 35. Consider the ordered Banach algebra $A_{0}=\mathbb{C}^{n}$, where $n \in \mathbb{N}$ and $n \geq 2$, under the natural algebraic operations, multiplication, and order and under some algebra-norm. Then the ordered Banach algebra $A$ obtained from $A_{0}$ by adjoining a unit gives the required example. Another example is the ordered Banach algebra $A=\mathbb{C}^{2}$ under the natural algebraic operations and order, the multiplication given by $\left(x_{1}, x_{2}\right)\left(y_{1}, y_{2}\right)=\left(x_{1} y_{1}, x_{1} y_{2}+x_{2} y_{1}\right)$, and the norm $\left\|\left(x_{1}, x_{2}\right)\right\|_{A}=\left|x_{1}\right|+\left|x_{2}\right|$. Indeed, as is easy to see, the element $\mathbf{e}=(1,0)$ is a unit of $A$, $\mathbf{O I}(A)=\{0, \mathbf{e}\}$, and the spectrum $\sigma(x ; A)=\left\{x_{1}\right\}$, where $x=\left(x_{1}, x_{2}\right)$. Then every element of the algebra $A$ is irreducible, $0 \leq(1,0)<(1,1)$, and $r((1,0))=r((1,1))=1$.

The situation does not change in the case of the algebra of the form $B(E)$. Indeed, let $H$ be an arbitrary real Hilbert space and let $z \in H$ with $\|z\|_{H}=1$. Under the order generated by the ice cream cone $K=\left\{y \in H:\langle y, z\rangle \geq \frac{1}{\sqrt{2}}\|y\|_{H}\right\}$, the space $H$ is a real ordered Banach space. Since the cone $K$ is generating, $B(H)$ is a real ordered Banach algebra and, hence, $B\left(H_{\mathbb{C}}\right)$ is a complex ordered Banach algebra, where $H_{\mathbb{C}}$ is the complexification of $H$. If $\operatorname{dim} H \geq 3$ then [5] the center $\left(B\left(H_{\mathbb{C}}\right)\right)_{I}=\{\lambda I: \lambda \in \mathbb{R}\}$ and, in particular, we have $\mathrm{OI}\left(B\left(H_{\mathbb{C}}\right)\right)=\{0, I\}$. There exists (see [5] once more) a non-zero positive operator $T$ satisfying $T^{2}=0$. Obviously, $I+T$ is an irreducible element of $B\left(H_{\mathbb{C}}\right), 0 \leq I<I+T$, and $r(I)=r(I+T)=1$. On the other hand, if $E$ is a two-dimensional ordered Banach space with generating cone $E^{+}$then, as is well known, $E^{+}$is a lattice cone. From the latter follows easily that the ordered Banach algebra $B(E)$ has a disjunctive product and, hence, Theorem 34 can be applied in this case.

An arbitrary Banach algebra $B$ with a unit is said to be Fredholm if the following three conditions hold: 
(a) The open subset $\Phi(B)$ of $B$ is determined and $\Phi(B)=-\Phi(B)$. Elements of $\Phi(B)$ are called Fredholm;

(b) Two functions nul, def $: B \rightarrow \mathbb{N} \cup\{0, \pm \infty\}$ are determined such that nul $b=\operatorname{nul}(-b)$ and $\operatorname{def} b=\operatorname{def}(-b)$ for all $b \in B$ and the set $\operatorname{Inv} B$ of invertible elements of $B$ satisfies $\operatorname{Inv} B=\{b \in B: \operatorname{nul} b=\operatorname{def} b=0\}$;

(c) The punctured neighbourhood property holds: if $b \in \Phi(B)$ then there exists a number $\epsilon>0$ such that nul $(\lambda-b)$ and $\operatorname{def}(\lambda-b)$ are constants on the set $\{\lambda \in \mathbb{C}: 0<|\lambda|<\epsilon\}$.

As is well known, the Banach algebra $B(Z)$, where $Z$ is an arbitrary Banach space, is Fredholm (see [1, Section 4.4]). In this case, $\Phi(B(Z))$ coincides with the class of all Fredholm operators on $Z$ and nul $T=\operatorname{dim} N(T)$ and $\operatorname{def} T=\operatorname{codim} R(T)$ for all $T \in \Phi(B(Z))$. Moreover, every Banach algebra $B$ with a unit $\mathbf{u}$ is Fredholm (see [ [8, Sections F2 anf F3]), i.e., the set $\Phi(B)$ and two functions nul and def on $B$ satisfying the required properties can be defined. Nevertheless, in [8] for the determination of these objects the notion of inessential ideal $J$ was used. That is, $J$ is an algebraic ideal and zero is the only possible accumulation point of $\sigma(b ; B)$ for each $b \in J$. In this case, the set $\Phi(B)$ is defined by

$$
\Phi(B)=\{b \in B: \mathbf{u}-a b, \mathbf{u}-b a \in J \text { for some } a \in B\} .
$$

In the definition of the Fredholm algebra given above the notion of inessential ideal is not required. Moreover, in some cases, e.g., of the algebra $C(K)$, where the compact space $K$ does not contain unisolated points, the zero ideal is a unique inessential ideal.

Theorem 36. Let an ordered Banach algebra $A$ satisfy Axiom $\left(\mathbf{A}_{1}\right)$ and let the cone $A^{+}$be normal. Let $a, b \in A$ be such that $0 \leq b<a$, the element $a$ is irreducible, $r(a)$ is a pole of $R(\cdot, a)$. Then each of the following conditions guarantees the inequality $r(b)<r(a)$ :

(a) $A$ is a Fredholm algebra and $r(a)-a \in \Phi(A)$;

(b) There exists an inessential ideal $J$ of $A$ which contains the residue $a_{-1}$;

(c) The ideal $\mathcal{F}(A)$ of finite-rank elements is inessential.

Proof. (a) Proceeding by contradiction, we assume $r(b)=r(a)$. For arbitrary $\epsilon \in[0,1)$, we define the element $a_{\epsilon}$ by $a_{\epsilon}=(1-\epsilon) a+\epsilon b$. Obviously, $0 \leq b \leq a_{\epsilon} \leq a$. Therefore, in view of the normality of the cone $A^{+}, r\left(a_{\epsilon}\right)=r(a)$. Moreover, $r(a)-a_{\epsilon} \rightarrow r(a)-a$ as $\epsilon \rightarrow 0$ and, hence, $r(a)-a_{\epsilon} \in \Phi(A)$ for sufficiently small $\epsilon$. Fix such a number $\epsilon$. Then $\operatorname{nul}\left(\lambda-a_{\epsilon}\right)$ and def $\left(\lambda-a_{\epsilon}\right)$ are constants on the set $\{\lambda \in \mathbb{C}: 0<|\lambda-r(a)|<\delta\}$ for some $\delta>0$. On the other hand, for $\lambda$ close to $r(a)$, we have the inclusion $\lambda-a \in \operatorname{Inv} A$ or nul $(\lambda-a)=\operatorname{def}(\lambda-a)=0$. Since the element $\lambda-a_{\epsilon}$ is invertible for $|\lambda|>r(a)$, the identities nul $\left(\lambda-a_{\epsilon}\right)=\operatorname{def}\left(\lambda-a_{\epsilon}\right)=0$ hold for $|\lambda|>r(a)$ and so for all numbers $\lambda$ satisfying $0<|\lambda-r(a)|<\delta$. Therefore, for such $\lambda$ the element $\lambda-a_{\epsilon}$ is invertible and, in particular, $r(a)$ is an isolated point of $\sigma\left(a_{\epsilon}\right)$. Using the normality cone $A^{+}$once more, we conclude that $r(a)$ is a simple pole of $R\left(\cdot, a_{\epsilon}\right)$. The residue $\left(a_{\epsilon}\right)_{-1}$ satisfies the relations

$$
r(a)\left(a_{\epsilon}\right)_{-1}=a_{\epsilon}\left(a_{\epsilon}\right)_{-1} \leq a\left(a_{\epsilon}\right)_{-1} \text { or } 0 \leq(a-r(a))\left(a_{\epsilon}\right)_{-1} .
$$


On the other hand, $a_{-1}(a-r(a))\left(a_{\epsilon}\right)_{-1}=0$. Taking into account the relation $a_{-1} \gg 0$, we get

$$
a\left(a_{\epsilon}\right)_{-1}=r(a)\left(a_{\epsilon}\right)_{-1}=a_{\epsilon}\left(a_{\epsilon}\right)_{-1}=((1-\epsilon) a+\epsilon b)\left(a_{\epsilon}\right)_{-1}
$$

and, hence, $(a-b)\left(a_{\epsilon}\right)_{-1}=0$. The element $a_{\epsilon}$ is also irreducible and so $\left(a_{\epsilon}\right)_{-1} \gg 0$. Now the last equality yields $a=b$, a contradiction.

(b) As was mentioned above, $A$ is a Fredholm algebra and the set $\Phi(A)$ of Fredholm elements can be defined via the formula (28). The coefficients $a_{-1}$ and $a_{0}$ of the Laurent series expansion of $R(\cdot, a)$ around $r(a)$ satisfies $\mathbf{e}-(r(a)-a) a_{0}=\mathbf{e}-a_{0}(r(a)-a)=a_{-1}$ and, hence, $r(a)-a \in \Phi(A)$. Now the required assertion follows at once from part (a).

(c) It suffices to observe the inclusion $a_{-1} \in \mathcal{F}(A)$ and to use part (b).

\section{The closedness of the center}

A normed algebra with a unit e and with a (closed, convex) cone $A^{+}$is called an ordered normed algebra [5] if $\mathbf{e} \geq 0$ and the inequalities $a, b \geq 0$ imply $a b \geq 0$. The center [5] of an ordered normed algebra $A$ is called the order ideal $A_{\text {e }}$ generated by e (see Example 2(b)), i.e.,

$$
A_{\mathbf{e}}=\left\{a \in A:-\lambda \mathbf{e} \leq a \leq \lambda \mathbf{e} \text { for some } \lambda \in \mathbb{R}^{+}\right\} .
$$

The aim of this section is to prove the closedness of the center $A_{\mathrm{e}}$ in $A$ (Theorem 38).

As was shown in [5], if an element $a \in A_{\mathbf{e}}$ then $a^{2} \geq 0$. The next result makes more precisely this fact.

Lemma 37. In an arbitrary ordered normed algebra $A$ the following identity holds

$$
A_{\mathbf{e}}=\left\{a \in A:(\lambda+a)^{2} \geq 0 \text { for every } \lambda \in \mathbb{R}\right\} .
$$

Proof. In view of the remarks above, it suffices to show that if $(\lambda+a)^{2} \in A^{+}$for all real $\lambda$ then $a \in A_{\mathbf{e}}$. To this end, we consider the Banach algebra $B$ being a completion of $A$ and $K=\overline{A^{+}}$, where the closure was taken in $B$. Obviously, $K$ is a wedge and $K \cdot K \subseteq K$. The relations $a^{2} \in A^{+}$and $\left(\lambda^{2}-a^{2}\right)^{2} \in A^{+}$with $\lambda \in \mathbb{R}$ are valid. For $\lambda>r(a)$ the element $\left(\lambda^{2}-a^{2}\right)^{-1}$ is well defined and belongs to $K$. Whence $\lambda^{2}-a^{2} \in A^{+} \cdot K \subseteq K$. On the other hand, $\lambda^{2}-a^{2} \in A$. In view of the closedness of $A^{+}$in $A, \lambda^{2}-a^{2} \in K \cap A=A^{+}$. Therefore, $0 \leq_{A^{+}} a^{2} \leq_{A^{+}} \leq \lambda^{2} \mathbf{e}$ and, in particular, $a^{2} \in A_{\mathbf{e}}$. Next, using the relation $(\mathbf{e} \pm a)^{2} \in A^{+}$, we obtain $\pm 2 a \leq_{A^{+}} \mathbf{e}+a^{2}$. Finally, $a \in A_{\mathbf{e}}$.

Theorem 38. The center $A_{\mathrm{e}}$ is a closed subset of an arbitrary ordered normed algebra $A$.

Proof. Consider a sequence $\left\{a_{n}\right\}$ in the center $A_{\mathbf{e}}$ satisfying $a_{n} \rightarrow a$ in $A$ as $n \rightarrow \infty$. Then $\left(\lambda+a_{n}\right)^{2} \geq 0$ for all $\lambda \in \mathbb{R}$ and, hence, $(\lambda+a)^{2} \geq 0$. In view of the preceding lemma, the element $a \in A_{\mathbf{e}}$.

The Minkowski norm $\|\cdot\|_{\mathrm{e}}$ can be defined on the center $A_{\mathrm{e}}$ (see (7)). Under this norm, $A_{\mathrm{e}}$ is a (real) ordered normed algebra. The next result follows immediately from the equality $A_{\mathbf{e}}=\bigcup_{n=1}^{\infty} n[-\mathbf{e}, \mathbf{e}]$, the Baire category theorem, and the preceding theorem.

Corollary 39. In an ordered Banach algebra $A$ the embedding $\left(A_{\mathbf{e}},\|\cdot\|_{A}\right) \rightarrow\left(A_{\mathbf{e}},\|\cdot\|_{\mathbf{e}}\right)$ is continuous. In particular, there exists a constant $c>0$ satisfying $\|a\|_{\mathbf{e}} \leq c\|a\|_{A}$ for all $a \in A_{\mathbf{e}}$. 


\section{References}

[1] Abramovich Y.A., Aliprantis C.D.: An invitation to operator theory. Graduate Studies in Mathematics, Vol. 50, American Mathematical Society, Providence, RI, USA, 2002.

[2] Alekhno E.A.: Spectral properties of band irreducible operators, Proceedings Positivity IV - Theory and Applications. Dresden (Germany), (2006), 5-14.

[3] Alekhno E.A.: Some properties of essential spectra of a positive operator. Positivity. 2007. — Vol. 11, No 3. - P. 375-386.

[4] Alekhno E.A.: The irreducibility in ordered Banach algebras. Positivity. — 2012. Vol. 16, No 1. - PP. 143-176.

[5] Alekhno E.A.: The order continuity in ordered algebras. To appear in Positivity.

[6] Aliprantis C.D., Burkinshaw O.: Positive operators. Springer, Dordrecht, Netherlands, 2006.

[7] Aliprantis C.D., Tourky R.: Cones and duality, Graduate Studies in Mathematics, Vol. 84, American Mathematical Society, Providence, RI, USA, 2007.

[8] Barnes B.A., Murphy G.J., Smyth M.R.F., West T.T..: Riesz and Fredholm theory in Banach algebras. Pitman, London, UK, 1982.

[9] Bonsall F.F., Duncan J.: Complete normed algebras. Springer, Berlin, Germany, 1973.

[10] Burger I., Grobler J.J.: Spectral properties of positive elements in Banach lattice algebras. Quaestiones Mathematicae. — 1995. — Vol. 18, No 1-3. — PP. 261-270.

[11] Glück J.: On the peripheral spectrum of positive operators. Positivity. - 2016. Vol. 20, No 2. - PP. 307-336.

[12] Kitover A.K., Wickstead A.W.: Operator norm limits of order continuous operators. Positivity. - 2005. - Vol. 9, No 2. - PP. 341-355.

[13] Mouton S., Raubenheimer H.: More spectral theory in ordered Banach algebras. Positivity. — 1997. — Vol. 1, No 4. — PP. 305-317.

[14] Niiro F., Sawashima I.: On the spectral properties of positive irreducible operators in an arbitrary Banach lattice and problems of H.H. Schaefer. Sci. Papers College Arts Sci. Univ. Tokyo. — 1966. — No 16. — PP. 145-183.

[15] Raubenheimer H., Rode S.: Cones in Banach algebras. Indag. Math., N.S. — 1996. — Vol. 7, No 4. - PP. 489-502.

[16] Schaefer H.H.: Banach lattices and positive operators. Springer, Berlin, Germany, 1974.

[17] Schaefer H.H.: On theorems of de Pagter and Ando-Krieger. Math. Z. — 1986. — Vol 192. - PP. 155-157. 